\title{
Combined Chemisorption and Complexation Generate siRNA Nanocarriers with Biophysics Optimized for Efficient Gene Knockdown and Air-Blood Barrier Crossing
}

Alexander Taschauer, Wolfram Polzer, Stefan Pöschl, Slavica Metz, Nathalie Tepe, Simon Decker, Norbert Cyran, Julia Scholda, Julia Maier, Hermann Bloß, Martina Anton, Thilo Hofmann, Manfred Ogris,* and Haider Sami*

Cite This: ACS Appl. Mater. Interfaces 2020, 12, 30095-30111

Read Online

ABSTRACT: Current nucleic acid (NA) nanotherapeutic approaches face challenges because of shortcomings such as limited control on loading efficiency, complex formulation procedure involving purification steps, low load of NA cargo per nanoparticle, endosomal trapping, and hampered release inside the cell. When combined, these factors significantly limit the amount of biologically active NA delivered per cell in vitro, delivered dosages in vivo for a prolonged biological effect, and the upscalability potential, thereby warranting early consideration in the design and developmental phase. Here, we report a versatile nanotherapeutic platform, termed auropolyplexes, for improved and efficient delivery of small interfering

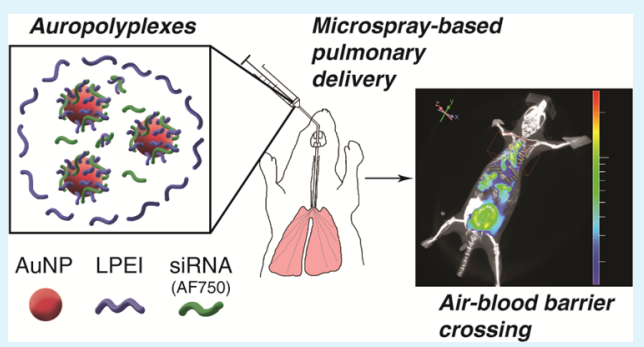
RNA (siRNA). Semitelechelic, thiolated linear polyethylenimine (PEI) was chemisorbed onto gold nanoparticles to endow them with positive charge. A simple two-step complexation method offers tunable loading of siRNA at concentrations relevant for in vivo studies and the flexibility for inclusion of multiple functionalities without any purification steps. SiRNA was electrostatically complexed with these cationic gold nanoparticles and further condensed with polycation or polyethyleneglycol-polycation conjugates. The resulting auropolyplexes ensured complete complexation of siRNA into nanoparticles with a high load of $\sim 15,500$ siRNA molecules/nanoparticle. After efficient internalization into the tumor cell, an $80 \%$ knockdown of the luciferase reporter gene was achieved. Auropolyplexes were applied intratracheally in Balb/c mice for pulmonary delivery, and their biodistribution were studied spatio-temporally and quantitatively by optical tomography. Auropolyplexes were well tolerated with $\sim 25 \%$ of the siRNA dose remaining in the lungs after 24 h. Importantly, siRNA was released from auropolyplexes in vivo and a fraction also crossed the air-blood barrier, which was then excreted via kidneys, whereas $>97 \%$ of gold nanoparticles were retained in the lung. Linear PEI-based auropolyplexes offer a combination of successful endosomal escape and better biocompatibility profile in vivo. Taken together, combined chemisorption and complexation endow auropolyplexes with crucial biophysical attributes, enabling a versatile and upscalable nanogold-based platform for siRNA delivery in vitro and in vivo.

KEYWORDS: gold nanoparticle, linear polyethylenimine, siRNA knockdown, pulmonary delivery, intratracheal, tomographic optical imaging, biodistribution

\section{INTRODUCTION}

Nanotechnology has the potential to overcome current challenges associated with nucleic acid-based therapeutics and is an active area of nanomedicine application. Preclinical and clinical translation of nucleic acid-based nanomedicines is decisively dependent on factors, including, but not limited to, loading efficiency, yield and scale-up, effective delivery to target organ/cells, and successful endosomal escape. ${ }^{1-3}$ For instance, high and tunable loading efficiency is indeed crucial when considered in the context of the "total nanoparticle load" needed to deliver the desired dosage in vivo for an efficacious/ prolonged biological effect ${ }^{3-5}$ and the toxicity associated with high nanoparticle loads in vivo. A high load of nucleic acid per nanoparticle is needed to account for delivery challenges owing to low cell uptake and low endosomal escape rates. ${ }^{3}$ Importantly, these crucial factors should be considered already during the optimization and development phase of formulations to ensure translation at later stages. ${ }^{5}$ Most of the existing nanoformulations for short nucleic acids, like short interfering RNA (siRNA), fall in one of the following categories: (i) layerby-layer (LBL) self-assembly to include siRNA as one of the

Received: April 10, 2020

Accepted: June 9, 2020

Published: June 9, 2020 


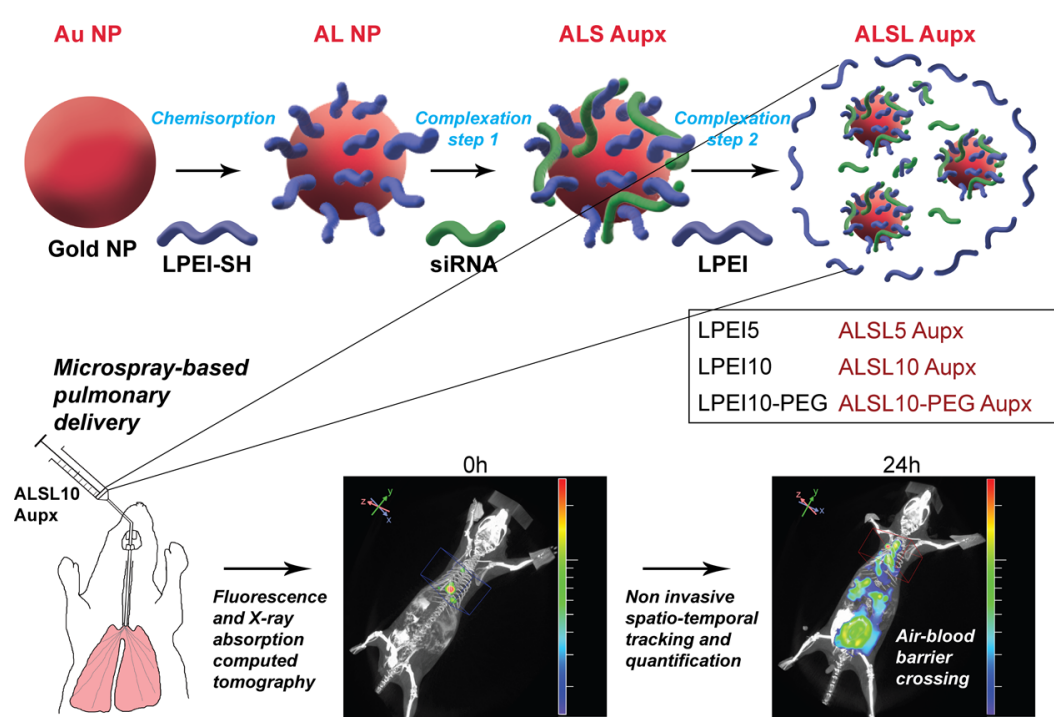

Figure 1. Schematic illustration of auropolyplex formulation and their pulmonary delivery by microspray-based aerosolization. Top: auropolyplex formulation involved combined chemisorption (of thiolated linear polyethylenimine [LPEI-SH] on gold nanoparticles [AuNP] to form cationic NPs $[\mathrm{AL} \mathrm{NP}]$ ) and complexation of these cationic NPs with siRNA (complexation step 1) and the desired LPEI polymer (complexation step 2; LPEI5 or LPEI10 or LPEI10-PEG) forming different versions of auropolyplexes [ALSL Aupx] with different functionalities, depending on the polymer used at the last step (inset box). Bottom: in vivo biodistribution of the near-infrared dye (AF750)-labeled siRNA-loaded ALSL10 auropolyplexes after microspray-based intratracheal administration was studied noninvasively and spatio-temporally by fluorescence imaging tomography (FLIT)/X-ray absorption computed tomography followed by AF750-siRNA and gold quantification by fluorescence-based absolute quantification and inductively coupled plasma mass spectrometry, respectively.

layers, (ii) encapsulation/conjugation of siRNA within/to the carrier system, and (iii) electrostatic complexation of siRNA with cationic moieties to form complexes (polyplexes, lipoplexes, etc.). In the case of LBL, despite the versatility and other advantages for designing nanosized vehicles for siRNA delivery, ${ }^{4,6}$ it is associated with challenges like low yield and repeated purification steps at each layer synthesis, thereby drastically limiting scale-up and control on siRNA loading efficiency. Additionally, in the case of LBL-coated nanoparticles (NPs), it is preferable to have a reduced number of layers, ${ }^{8,9}$ which further limits the total amount of loaded siRNA. Within the second category, siRNA can be encapsulated within lipid-based nanoparticles, which are considered clinically relevant and potent delivery systems ${ }^{10}$ or chemically conjugated to the nanocarrier, polymer, or ligand. ${ }^{11}$ The chemisorption of thiolated siRNA directly onto the surface of gold nanoparticles to form spherical nucleic acids is a very promising approach. ${ }^{12}$ However, in this case, intracellular release of siRNA can be limiting as it has been observed that thiolated nucleic acid is still bound to the gold NP surface after delivery into cells, ${ }^{13}$ hence limiting access to the cytoplasmic transcriptional machinery. Polyplexes can overcome such limitations by offering desirable yields, scalability, ${ }^{14}$ and control on loading efficiency as all of the nucleic acid are complexed. Also, polyplexes can be generated at high concentrations for in vivo applications ${ }^{14}$ and enable multiple functionalizations with shielding domains (like polyethylene glycol, targeting ligands, and endosomolytic agents $)^{15}$ for preclinical and clinical relevance. The very crucial step of endosomal escape is achieved with polymers like polyethylenimine (PEI), both in linear (LPEI) or branched (BPEI) formats. For siRNA knockdown studies, BPEI-based polyplexes often show better results in comparison to LPEI. ${ }^{16}$ However, BPEI-based formulations limit the allowed treatment dose in vivo owing to its acute toxicity. ${ }^{17}$ LPEI is preferable due to a more biocompatible profile, ${ }^{18}$ although this also highly depends on the molecular weight used. ${ }^{19}$ Considering these current approaches and their associated challenges, it is desirable to have formulations that can address these limitations so as to deliver the required amounts of siRNA to the target organ. LPEI is available in GMP quality and is being currently used in several clinical trials, ${ }^{20-22}$ thus making LPEI-based formulations attractive for offering a successful endosomal escape and better biocompatibility profile in vivo. In vivo imaging can significantly assist in the development of nucleic acid-based therapies ${ }^{23}$ by deciphering important delivery and therapy concepts like quantification of retention in the target organ, biodistribution events, transfection efficiency, etc. This helps in optimizing delivery agents and their routes of administration and also identifying bottlenecks. We have established an imaging-based method for spatiotemporal tracking of siRNA in vivo by near-infrared fluorescence imaging, along with fluorescence imaging (FLIT) and X-ray absorption computed tomography (CT)..$^{24,25}$

The present study combines the advantages of the electrostatic complexation approach with chemisorption on gold nanoparticles to develop an LPEI-based novel formulation, termed as auropolyplexes, for efficient siRNA formulation and delivery in vitro and in vivo (Figure 1). Toward this, gold nanoparticles were functionalized with thiolated LPEI to make a cationic nanocarrier and used for complexing with siRNA to generate auropolyplexes by a facile and convenient two-step complexation procedure, as shown in Figure 1. This ensures complexation of total siRNA, as confirmed by gel retardation studies, thereby giving precise control on loading efficiency. The second complexation step is for inclusion of excess LPEI to provide enough cationic charge for sufficient cell uptake and appropriate endosomal escape rate as these are crucial ratelimiting steps in siRNA delivery. ${ }^{3,26}$ The additional complexation step endows flexibility and tailorability to the formulation approach as PEGylated LPEI (instead of LPEI) was used at the 
second complexation step to provide "stealth" attributes for potential in vivo applications. After intratracheal microspraying of auropolyplexes, optical tomography-based (i) noninvasive spatio-temporal tracking and (ii) absolute quantification of AF750-siRNA delivered in vivo (within different organs) were attempted to study the amounts available for potential therapeutic effects (Figure 1). The gold amount was investigated in organs by inductively coupled plasma mass spectrometry to compare with siRNA's biodistribution so as to decipher in vivo release of siRNA from the auropolyplexes. This work presents auropolyplexes as a facile and versatile nanoformulation with tunable siRNA loading for both in vitro and in vivo experiments and superior gene knockdown attributes, which has the potential to be an efficacious multifunctional nanomedicine platform for siRNA delivery.

\section{MATERIALS AND METHODS}

Materials. All siRNA samples used in this study were kindly supplied by GlaxoSmithKline (GSK; UK). The siRNA for the knockdown of firefly luciferase (anti-luc siRNA) with the sequence 5 'CUUACGCUGAGUACUUCGAdTdT- $3^{\prime}$ (sense strand) has two phosphorothioate bonds on the $3^{\prime}$ terminus of the sense strand and one $3^{\prime}$ terminal phosphorothioate bond on the antisense strand $\left(M_{w}\right.$ $($ duplex $)=14,384 \mathrm{Da})$. The siRNA used as a negative control (control siRNA) with the sequence 5'-AUCGUACGUACCGUCGUAUdTdT-3' (sense strand) shows two phosphorothioate bonds on the $3^{\prime}$ terminus of both the sense and antisense strand $\left(M_{\mathrm{w}}\right.$ $($ duplex $)=14,468 \mathrm{Da})$. Labeled negative control siRNA was generated by coupling AF647 or AF750 to the 3' terminus of the sense strand using $\mathrm{N}$-hydroxysuccinimide (NHS)-based chemistry. In the following sections, labeled siRNA is described as AF647-siRNA and AF750-siRNA. OPSS-PEG-NHS (3-(2-pyridyldithio)propionamide-PEG- $N$-hydroxysuccinimide ester $)\left(M_{\mathrm{w}}=2000 \mathrm{Da}\right)$ was purchased from Rapp Polymere (Germany). All further compounds used for synthesis, for physicochemical, and for biological evaluation were purchased from Sigma Aldrich (Austria). All polymer and buffer solutions were filtered through $0.22 \mu \mathrm{m}$ cellulose acetate filters prior to use; polymer solutions were quantified after filtration. Water was purified with a Sartorius AriumPro system (Germany). TrypLE Express for cell detachment was purchased from Thermo Fisher Scientific (Germany), and passive lysis buffer was purchased from Promega (Germany).

Methods. Synthesis of LPEI.HCI (Linear Polyethylenimine as $\mathrm{HCl}$ Salt). Both $\alpha$-benzyl- $\omega$-thiol LPEI $\left(M_{\mathrm{n}}=5000 \mathrm{Da}\right) \cdot \mathrm{HCl}$ and $\alpha$ methyl- $\omega$-hydroxy LPEI $\left(M_{\mathrm{w}}=10,000 \mathrm{Da}\right) \cdot \mathrm{HCl}$ were synthesized based on a previously described method. ${ }^{27}$ The molecular weights stated refer to the free base of the respective polymer. Briefly, $2 \mathrm{~g}$ of either $\alpha$-benzyl- $\omega$-thiol poly(2-ethyl-2-oxazoline) or $\alpha$-methyl- $\omega$ hydroxy poly(2-ethyl-2-oxazoline) was dissolved in $50 \mathrm{~mL}$ of $\mathrm{HCl}$ $(7 \mathrm{M})$ and heated under reflux for $16 \mathrm{~h}$. $\alpha$-Benzyl- $\omega$-thiol LPEI-HCl (denoted as LPEI5) or $\alpha$-methyl- $\omega$-hydroxy LPEI-HCl (denoted as LPEI10) formed a white precipitate, which was further purified by centrifugation. After three washing steps with $7 \mathrm{M} \mathrm{HCl}$, the precipitate was dissolved in $100 \mathrm{~mL}$ of water and lyophilized. The quality control of compounds dissolved in $\mathrm{D}_{2} \mathrm{O}$ was conducted by ${ }^{1} \mathrm{H}$ NMR on a Bruker Avance $200 \mathrm{MHz}$ system (Bruker, Billerica, USA). Chemical shifts $(\delta)$ expressed in parts per million $(\mathrm{ppm})$ were analyzed using the peak derived from the solvent as reference. In the case of $\alpha$-benzyl- $\omega$-thiol LPEI-HCl (LPEI5; thiol terminated LPEI), the ratio between LPEI and free thiol groups was evaluated by copper and Ellman's assay, as described. ${ }^{27,28}$ LPEI5. $\mathrm{HCl}$ was stored for long term under dry conditions at room temperature to reduce the probability of thiol oxidation. Stock solutions in water were stored at $-20{ }^{\circ} \mathrm{C}$ for not longer than 2 months where no decrease of the free thiol content could be detected.

Synthesis of LPEI $\left(M_{w}=10,000\right.$ Da)-PEG $\left(M_{w}=2000\right.$ Da $)$ Cysteine Conjugate (LPEI10-PEG). Conjugation of PEG to LPEI was conducted based on the protocol by Schaffert et al. ${ }^{29}$ In brief, $1 \mathrm{~g}$ of
LPEI10. $\mathrm{HCl}$ was suspended in $20 \mathrm{~mL}$ of $\mathrm{NaOH}(1 \mathrm{M})$. LPEI10 precipitated as a free base. The mixture was heated under reflux, and $\mathrm{NaOH}(1 \mathrm{M})$ was added in small portions until LPEI10 was entirely dissolved. After cooling down to room temperature, the white precipitate was purified by centrifugation and washed with $1 \mathrm{M}$ $\mathrm{NaOH}$ and water. LPEI10 was resuspended in $30 \mathrm{~mL}$ of water and lyophilized. For PEGylation, LPEI10 was dissolved in $1.5 \mathrm{~mL}$ of dry ethanol, mixed with $100 \mu \mathrm{L}$ of NHS-PEG-OPSS (two equivalents based on LPEI) in dry dimethyl sulfoxide, and incubated for $3 \mathrm{~h}$ at 35 ${ }^{\circ} \mathrm{C}$ under continuous mixing. The reaction was quenched with $100 \mu \mathrm{L}$ of Tris $\mathrm{HCl}(1 \mathrm{M} ; \mathrm{pH} 8.0)$. The product was purified by ion exchange chromatography (column HR10/10, MacroPrep High S; BioRad, US) using an ÄktaPure system (GE Healthcare, Germany), applying a $\mathrm{NaCl}$ gradient of $0.5-3 \mathrm{M}$ in $20 \mathrm{mM}$ aqueous HEPES solution $(\mathrm{pH}$ 7.4). Fractions containing LPEI-PEG-OPSS were pooled, dialyzed against water for $24 \mathrm{~h}$, and lyophilized. Thereafter, LPEI-PEG-OPSS was dissolved in $2 \mathrm{~mL}$ of $20 \mathrm{mM}$ HEPES/10\% acetonitrile ( $\mathrm{pH}$ 7.4; degassed) and mixed with a solution of L-cysteine (five equivalents based on OPSS) in $20 \mathrm{mM}$ HEPES/10\% acetonitrile ( $\mathrm{pH}$ 7.4; degassed). The reaction was monitored by analyzing the amount of released 2-thiopyridone (absorption maximum at $343 \mathrm{~nm}$ ) by UV-vis spectrophotometry every $30 \mathrm{~min}$ until no change in the absorption could be detected. The conjugate was purified by ion exchange chromatography, dialyzed, and lyophilized, as described above. LPEI10-PEG was analyzed by ${ }^{1} \mathrm{H}$ NMR on a Bruker Avance 200 $\mathrm{MHz}$ system. The PEGylation degree was evaluated, correlating the integral of the PEG signal $\left[\mathrm{CH}_{2}-\mathrm{CH}_{2}-\mathrm{O} ; \delta(\mathrm{ppm})=3.72\right]$ with the integral of the LPEI signal $\left[\mathrm{CH}_{2}-\mathrm{CH}_{2}-\mathrm{N} ; \delta(\mathrm{ppm})=3.06\right]$.

Synthesis of Cationic Gold Nanoparticles. The synthesis of gold nanoparticles was conducted based on the modified procedure by Frens. ${ }^{30,31}$ For this, $25 \mathrm{~mL}$ of a $0.01 \%(\mathrm{w} / \mathrm{v}) \mathrm{HAuCl}_{4}$ solution in water was heated to $100{ }^{\circ} \mathrm{C}$ and then mixed with $180 \mu \mathrm{L}$ of an aqueous sodium citrate solution $(1 \%[\mathrm{w} / \mathrm{v}])$. The color of the solution changed gradually from faint yellow to red. The reaction mixture was stirred at $100{ }^{\circ} \mathrm{C}$ until no further color change could be observed. After cooling down to room temperature, the product quality was evaluated by analyzing the particle size by nanoparticle tracking analysis (NTA) with a NanoSight NS500 system (Malvern, UK). The solution was kept at room temperature until further usage. For surface functionalization of gold nanoparticles (AuNP) with LPEI5 (thiol terminated LPEI), the AuNP solution was adjusted to $\mathrm{pH} 8$ by adding small portions $(3-5 \mu \mathrm{L})$ of $\mathrm{NaOH}(1 \mathrm{M})$ under vigorous stirring. Thereafter, a stock solution of LPEI5 (thiol terminated LPEI) dissolved in water $(\mathrm{pH} 8)$ was added under constant stirring to obtain a final LPEIS concentration of $0.229 \mathrm{mM}$. This mixture was incubated at room temperature for $72 \mathrm{~h}$. Changes in the AuNP-derived SPR before and after coating was analyzed by UVvis spectrophotometry of the undiluted samples. For purification, 18 $\mathrm{mL}$ of this solution was centrifuged in $1 \mathrm{~mL}$ of aliquots for $10 \mathrm{~min}$ at $5000 \mathrm{~g}$ and $4{ }^{\circ} \mathrm{C}$ and washed with $1 \mathrm{~mL}$ of water per aliquot till there was no free LPEI detected in the supernatant, applying the copper assay. ${ }^{28}$ Finally, pellets were pooled and reconstituted in water with a final volume of $400 \mu \mathrm{L}$. The resulting cationic AuNPs are referred to as "AL NP". Nanoparticles were stored until further usage at room temperature for up to 1 week.

Auropolyplex Generation. For in vitro experiments, complexation was conducted at a final siRNA concentration of $10 \mu \mathrm{g} / \mathrm{mL}$ using water as a diluent for siRNA and LPEI, whereas for in vivo experiments, complexation was conducted at a final siRNA concentration of $133 \mu \mathrm{g} / \mathrm{mL}$ using HBG (HEPES buffered glucose; $20 \mathrm{mM}$ HEPES $/ 5 \%$ glucose at $\mathrm{pH}$ 7.4) as the diluent. For auropolyplex synthesis, equal volumes of a solution containing siRNA (conc. [in vitro], $40 \mu \mathrm{g} / \mathrm{mL}$ siRNA; conc. [in vivo], $533 \mu \mathrm{g} /$ $\mathrm{mL}$ ) and AL NP stock (described above) were mixed by flash pipetting in complexation step 1 . The mixture of AL NP with siRNA is termed "ALS Aupx". After an incubation for $45 \mathrm{~min}$ at room temperature, equal volumes of "ALS" and a solution containing LPEI (conc. [in vitro], $10 \mu \mathrm{g} / \mathrm{mL}$; conc. [in vivo], $267 \mu \mathrm{g} / \mathrm{mL}$ ) were mixed by flash pipetting in complexation step 2 . The mixture was again incubated for $45 \mathrm{~min}$ at room temperature until further usage. The 
resulting solution is described as following ALSL auropolyplexes (Aupxs)-“ALSL5" (for LPEI5 as the last layer), "ALSL10" (for LPEI10 as the last layer), or "ALSL10-PEG" (for LPEI10-PEG as the last layer). PEGylated auropolyplexes (ALSL10-PEG) were prepared at a final siRNA concentration of $133 \mu \mathrm{g} / \mathrm{mL}$ using HBG. To reach the same final siRNA and AL concentration, a 1:2 dilution of ALS was used as ALS Aupxs both for physicochemical and for in vitro evaluation of components. As polymer control treatments (i.e., without gold nanoparticles), the desired amount of siRNA (same as in the corresponding auropolyplex) was mixed with corresponding polymers LPEI5 or LPEI10 and was denoted as LPEI5 polymer + siRNA control or LPEI10 polymer + siRNA control, respectively. This was achieved by mixing "only LPEI" with siRNA in the same amounts, as used in complexation step 2 of corresponding auropolyplex synthesis, but without gold nanoparticles. In brief, equal volumes of a siRNA $(20 \mu \mathrm{g} / \mathrm{mL})$ and a LPEI (LPEI5 or LPEI10; LPEI concentration was the same as for complexation step 2 of auropolyplex generation) containing solution were mixed at a N/P ratio of 4 by flash pipetting. The incubation time was kept the same as for complexation step 2 of auropolyplex generation. These complexes were then used as polymer control treatments along with other treatments of ALS and ALSL Aupxs for transmission electron microscopy, cell association by flow cytometry, and gene knockdown studies.

Gel Retardation Assay. The gel retardation assay was done to study siRNA complexation at siRNA concentrations relevant for in vitro $(10 \mu \mathrm{g} / \mathrm{mL})$ and in vivo studies $(133 \mu \mathrm{g} / \mathrm{mL})$. Auropolyplexes were generated using different concentrations of siRNA (antiluciferase siRNA or AF750-siRNA) and different amounts of LPEI (LPEI5 or LPEI10). For an in vitro setup, auropolyplexes with antiluciferase siRNA and LPEI5 were prepared in water, reaching a final siRNA concentration of $10 \mu \mathrm{g} / \mathrm{mL}$. For an in vivo setup, AF750siRNA and LPEI10 were prepared in HBG at a final siRNA concentration of $133 \mu \mathrm{g} / \mathrm{mL}$. Free or complexed siRNA (200 ng) was loaded onto a $1.5 \%$ agarose gel sodium borate buffer $(\mathrm{pH} 8)$ together with one-sixth volume of $60 \%(\mathrm{v} / \mathrm{v})$ glycerol as loading buffer. For nucleic acid, staining ethidium bromide (EtBr) was used at a concentration of $0.5 \mu \mathrm{g} / \mathrm{mL}$. Gel electrophoresis was carried out in sodium borate buffer $(\mathrm{pH} 8)$ at $80 \mathrm{~V}$ for $60 \mathrm{~min}$. Imaging was conducted on a ChemiDoc MP system (Biorad, Vienna, Austria).

Nanoparticle Tracking Analysis (NTA). Size and $\zeta$-potential measurement by NTA was carried out in principle, as described. ${ }^{14}$ For size and concentration measurements, nanoparticles were diluted in the medium used for synthesis (water or HBG) for $\zeta$-potential measurements in $2.5 \mathrm{mM} \mathrm{NaCl}$. A dilution factor was chosen to obtain a particle concentration of $10^{8}$ to $10^{9}$ nanoparticles $/ \mathrm{mL}$, which correlates to 10-100 nanoparticles per frame. For size measurement, five videos with a duration of $60 \mathrm{~s}$ were acquired. In the case of $\zeta$ potential measurement, capture duration was $90 \mathrm{~s}$ and secondary duration was $30 \mathrm{~s}$. The $\zeta$-potential was measured applying $24 \mathrm{~V}$. For evaluating the $\zeta$-potential of nanoparticles, only measurements with a coefficient of correlation of at least 0.95 were considered for further analysis. AL samples were analyzed by NTA after purification (by centrifugation), while all other samples were analyzed directly without purification. NTA-based characterization of auropolyplex formulation was performed at each step for the final auropolyplexes prepared at siRNA concentrations of 10 and $133 \mu \mathrm{g} / \mathrm{mL}$, relevant for in vitro and in vivo studies, respectively.

The number of siRNA molecules per auropolyplex nanoparticle was estimated based on the gel retardation (giving the total amount of complexed/loaded siRNA in the auropolyplex formulation) and NTA data (giving the concentration of auropolyplex nanoparticles in the formulation). First, the total amount of siRNA loaded in the auropolyplex formulation was calculated from gel retardation studies for total complexation of siRNA. Second, the total number of nanoparticles in the auropolyplex formulation was measured by the nanoparticle tracking analysis while keeping the concentration in the prescribed range, as per NTA measurement specifications. Then, the total siRNA amount was divided by the total number of nanoparticles to calculate the number of siRNA molecules per auropolyplex nanoparticle.

TEM (Transmission Electron Microscopy). For TEM, every formulation was prepared in water, as described above. The undiluted sample $(5 \mu \mathrm{L})$ was deposited onto formvar-coated copper grids and dried overnight at room temperature. Imaging was done on a Libra 120 system (Zeiss, Germany) with a $\mathrm{LaB}^{6}$ filament and in column filter operating at $120 \mathrm{kV}$ using a bottom mount camera Sharp:eye TRS $(2 \times 2 \mathrm{k})$ and an in column camera Morada G2 (11 MP). Magnifications ranged from 8000- to 200,000-fold.

Cell Culture and Transduction. MDA-MB-231 human breast cancer cells (ATCC HTP-26) were cultured in DMEM (Dulbecco's modified Eagle medium) supplemented with $10 \%$ fetal bovine serum, L-glutamine, and antibiotics (penicillin/streptomycin) and lentivirally transduced with PGK-EGFPLuc, as described previously, ${ }^{32}$ to obtain MDA-MB-231Luc cells, which express the EGFP-luciferase (EGFPLuc) fusion protein under a constitutively active phosphoglycerol promoter PGK. EGFP-positive cells were sorted using a BD FACSAria II cell sorter (Becton Dickinson, US). MDA-MB-231Luc cells were used for all cell culture experiments.

Flow Cytometry and In Vitro Biocompatibility. MDA-MB-231Luc cells per well $\left(5 \times 10^{4}\right)$ were seeded into a transparent 96 -well plate $24 \mathrm{~h}$ prior to treatment. All formulations were prepared using AF647siRNA and added to cells at 2 or $4 \mu \mathrm{g} / \mathrm{mL}$ (based on siRNA) in a basal DMEM high glucose medium (without serum) for $4 \mathrm{~h}$. The medium supplemented with $10 \%$ fetal bovine serum, L-glutamine, and antibiotics (penicillin/streptomycin) was added after the above $4 \mathrm{~h}$ incubation step, and the cells were left in contact with different formulations in a serum containing medium for $20 \mathrm{~h}$. After a total of $24 \mathrm{~h}$ of uptake, cells were washed twice with Dulbecco's phosphatebuffered saline (DPBS), trypsinized with TrypLE Express, as per the manufacturer's instructions, resuspended in DPBS, and transferred into a polymerase chain reaction (PCR) plate (Nerbe, \#04-083-0150; Germany). Polyplexes based on branched polyethylenimine (BPEI; $M_{\mathrm{w}}=25,000 \mathrm{Da}$ ) were used as an internal positive control for cell binding studies at N/P 10 in HBS (HEPES buffered saline; $20 \mathrm{mM}$ HEPES $/ 150 \mathrm{mM} \mathrm{NaCl}$ at $\mathrm{pH}$ 7.4). Plates were kept at $4{ }^{\circ} \mathrm{C}$ for the whole measurement using an Inheco CPAC cooling unit (INHECO, Germany) when analyzed on a MacsQuant Analyzer 10 (Miltenyi Biotec, Bergisch-Gladbach, Germany). A live/dead assay was performed with DAPI staining and measured the live/dead cells by flow cytometry for investigating the biocompatibility behavior of auropolyplexes after $24 \mathrm{~h}$ of treatment, as done for cell binding studies. Percentage viability for each formulation was calculated based on untreated cells. For live/dead analysis, DAPI was used at a concentration of $1 \mu \mathrm{g} / \mathrm{mL}$ ( $405 \mathrm{~nm}$ excitation; $450 / 50 \mathrm{~nm}$ band pass emission filter). In total, 15,000 gated live cells were analyzed per well. The AF647 signal was acquired in the R1 channel ( $635 \mathrm{~nm}$ excitation; 655-730 nm band pass emission filter). Data were analyzed using FlowJo 10.1r5 (FlowJo LLC, Ashland, OR 97520, USA).

Binding/Uptake Analysis by CLSM (Confocal Laser Scanning Microscopy). MDA-MB-231Luc cells per well $\left(5 \times 10^{4}\right)$ were seeded into chamber slides (Nunc Lab-Tek II eight-well slides; Thermo Fisher Scientific, Germany) $24 \mathrm{~h}$ before treatment. Cells were incubated with formulations based on AF647-labeled siRNA ( $4 \mu \mathrm{g}$ / $\mathrm{mL}$ ) and further treated, as described above for flow cytometry. Polyplexes based on branched polyethylenimine (BPEI; $M_{\mathrm{w}}=25,000$ $\mathrm{Da}$ ) were used as positive control for cell binding/uptake studies at $\mathrm{N} / \mathrm{P} 10$ in HBS (HEPES buffered saline; $20 \mathrm{mM}$ HEPES/150 mM $\mathrm{NaCl}$ at $\mathrm{pH}$ 7.4). After $24 \mathrm{~h}$, cells were washed thoroughly with DPBS and fixed with $4 \%$ formaldehyde in HBS ( $\mathrm{pH} 7.4$ ) for $30 \mathrm{~min}$ at room temperature. Cell nuclei were stained with DAPI $(2 \mu \mathrm{g} / \mathrm{mL})$ and samples mounted with a Vectashield antifade mounting medium. Image acquisition was performed on a Leica TCS SPE microscope (Leica, Wetzlar, Germany) with a $63 \times$ oil immersion objective (NA 1.3) using a $405 \mathrm{~nm}$ laser excitation for DAPI, $488 \mathrm{~nm}$ for EGFP, and $635 \mathrm{~nm}$ for AF647. Emission ranges were tuned for optimal collection of emitted light, as per the respective fluorophore. Z-scans were conducted with a vertical resolution of $0.1 \mu \mathrm{m}$. Differential interference contrast (DIC) was used for transmitted light pictures. 
A

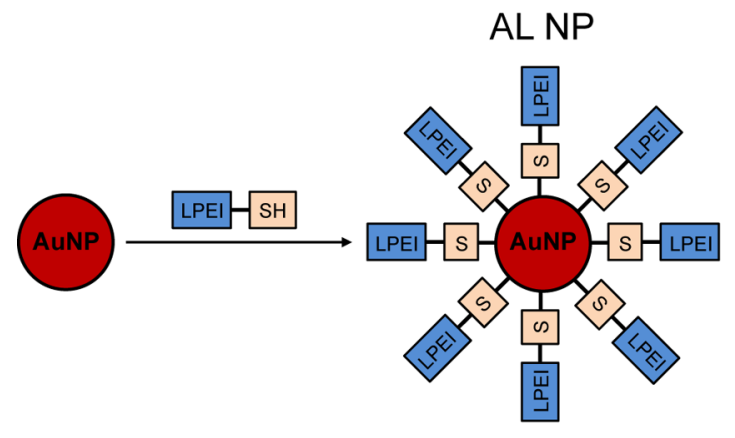

B

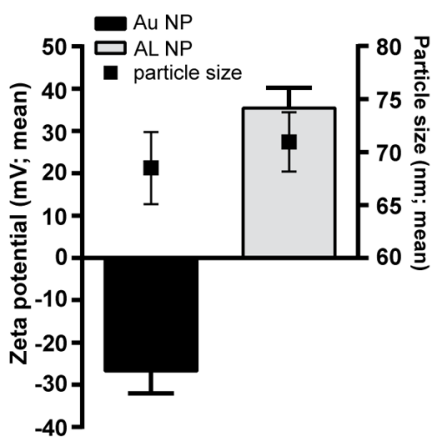

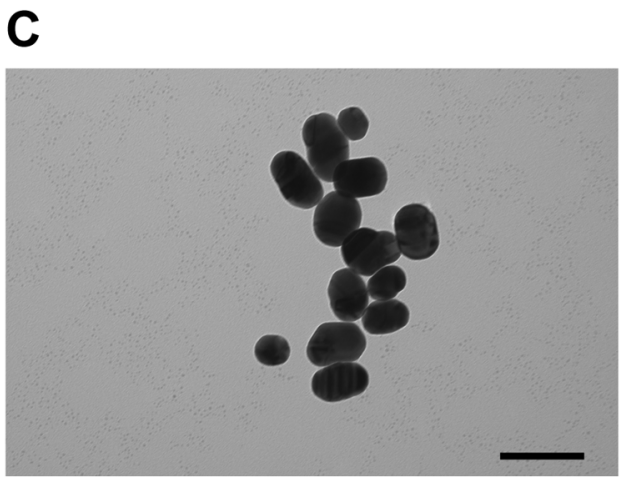

D

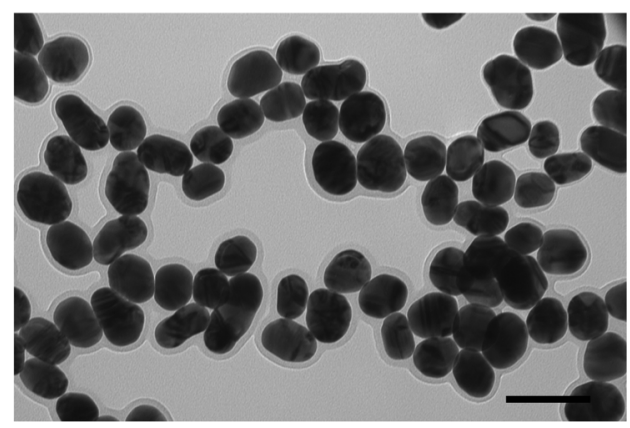

Figure 2. Synthesis and characterization of cationic gold nanoparticles. (A) Schematic illustration of the synthesis of cationic gold nanoparticles (AL NP) by chemisorption of thiol terminated linear polyethylenimine (LPEI5) on gold nanoparticles (AuNP). (B) Nanoparticle tracking analysisbased characterization of AuNP and AL NP for the $\zeta$-potential (bars, left $y$ axis) and particle size (boxes, right $y$ axis). (C, D) TEM micrographs of (C) AuNPs and (D) AL NPs. Scale bars, $100 \mathrm{~nm}$.

Further analysis of the acquired pictures was done with LasX software version 3.1.2.16221 (Leica).

In Vitro Gene Knockdown by the Firefly Luciferase Assay. MDAMB-231Luc cells per well $\left(2 \times 10^{4}\right)$ were seeded into a transparent 96-well plate $24 \mathrm{~h}$ before treatment. All formulations were prepared using anti-luc or control siRNA and added to cells at 2 or $4 \mu \mathrm{g} / \mathrm{mL}$ (based on siRNA) and further treated, as described above for flow cytometry. Polyplexes based on branched polyethylenimine (BPEI; $M_{\mathrm{w}}=25,000 \mathrm{Da}$ ) were used as positive control for knockdown studies at N/P 10 in HBS (HEPES buffered saline; $20 \mathrm{mM}$ HEPES/150 mM $\mathrm{NaCl}$ at pH 7.4). After a total of $48 \mathrm{~h}$, cells were washed with DPBS and lysed with $30 \mu \mathrm{L}$ of passive lysis buffer. The lysate $(10 \mu \mathrm{L})$ was analyzed for luciferase activity using a luciferase assay reagent (LAR). ${ }^{33}$ Luminescence was measured using an Infinite M200 system (Tecan, Grödig, Austria).

In Vivo Biodistribution by Fluorescence Imaging Tomography (FLIT) and X-ray Absorption Computed Tomography (CT). All animal procedures were approved by the local ethics committee and are in accordance with the Austrian law for the protection of animals and the EU directive 2010/63/EU. Female Balb/cJRj mice (Janvier Labs, Le-Genest, France) were housed under specified pathogen free (SPF) conditions in individually ventilated cages (Type $2 \mathrm{~L}$, Tecniplast, Hohenpeißenberg, Germany) with food and water ad libitum and a 12/12 h light/dark cycle. Mice were kept on a low fluorescent diet (AIN-76A; Brogaarden Korn \& Foder ApS, Denmark) for at least 10 days before treatment. ALSL10 auropolyplexes containing a total of $10 \mu \mathrm{g}$ of AF750-siRNA in HBG $(133 \mu \mathrm{g} / \mathrm{mL})$ were applied by intratracheal administration using a Microsprayer/syringe assembly (MSA-250-M; PennCentury, Inc., USA). Before administration to animals, the influence of the microspraying process on auropolyplexes was analyzed by NTA. Animals were imaged on an IVIS Spectrum CT system (PerkinElmer, USA) immediately after drug administration $(0 \mathrm{~h})$ and $24 \mathrm{~h}$ thereafter for $2 \mathrm{D}$ epifluorescence imaging and fluorescence imaging tomog-
raphy/X-ray absorption computed tomography (FLIT/CT) imaging, as described. ${ }^{24} 2 \mathrm{D}$ imaging was conducted in epifluorescence mode using the following excitation (Ex) and emission (Em) filter settings: Ex of $640 \mathrm{~nm}$ with Em of 680, 700, 720, 740, and $760 \mathrm{~nm}$ and Ex of $675 \mathrm{~nm}$ with Em of 720, 740, 760, 780, and $800 \mathrm{~nm}$. To ensure proper background subtraction, treated and untreated animals were imaged side by side. For 3D FLIT/CT imaging, the following fluorescence filter settings were used in transillumination mode: Ex of $675 \mathrm{~nm}$ (30 $\mathrm{nm} \mathrm{BW})$ and Em of $720 \mathrm{~nm}(20 \mathrm{~nm} \mathrm{BW})$. A 1:1.5 mixture of Scanlux $(300 \mu \mathrm{L})$ (active component: Iopamidol [300 $\mathrm{mg}$ iodine $/ \mathrm{mL}$ ]; Sanochemia, Vienna, Austria) with $5 \%$ glucose was used as a CT contrast agent. After the last imaging session, animals were sacrificed and ex vivo organ imaging was done in $2 \mathrm{D}$ epifluorescence mode using the settings described above but without spectral unmixing. The lungs, liver, spleen, kidneys, and stomach were stored at $-80^{\circ} \mathrm{C}$ until further analysis by ICP-MS.

Image analysis of $2 \mathrm{D}$ epifluorescence and FLIT/CT imaging data was done with Living Image software, as described in detail elsewhere. ${ }^{24}$ For $2 \mathrm{D}$ epifluorescence imaging data, background auto fluorescence (from animals) was distinguished from AF750 fluorescence by a spectral unmixing method. FLIT/CT imaging data was reconstructed to generate a virtual surface, which was then used for 3D analysis, enabling sagittal, coronal, and axial views. Absolute quantification of fluorescence signals within animals was done by placing 3D cuboid ROIs in areas covering the lungs, kidneys, and bladder and employing a fluorescence database for AF750-siRNA to quantify the signal in total pmol amount per ROI. This amount in pmol was estimated for all animals and average values plotted for different time points and different organs of interest.

Inductively Coupled Plasma-Mass Spectrometry (ICP-MS). The lungs, liver, spleen, kidneys, and stomach were cut into $3-5 \mathrm{~mm}$ fragments and digested in $8 \mathrm{~mL}$ of inverse aqua regia at $100{ }^{\circ} \mathrm{C}$ for 1 h. Each sample was then treated with $15 \mathrm{~mL}$ of $\mathrm{HNO}_{3}$ concentration at $180{ }^{\circ} \mathrm{C}$ for one further hour. For dissolving gold nanoparticles, 10 
$\mathrm{mL}$ of aqua regia was added and the mixture was incubated at $100{ }^{\circ} \mathrm{C}$ for $1 \mathrm{~h}$. All samples were finally diluted to a total mass of $40 \mathrm{~g}$ with purified water. Samples prepared following the same procedure without any tissue were used as blank for ICP-MS measurements. Total dissolved gold concentration $\left({ }^{197} \mathrm{Au}\right)$ measurements were conducted with a 7900 ICP-MS unit (Agilent, US), and ${ }^{209} \mathrm{Bi}$ was used as the internal standard. To evaluate total dissolved gold concentrations, the ICP-MS was calibrated with dissolved gold standards ranging from $5 \mathrm{ng} / \mathrm{L}$ to $50 \mu \mathrm{g} / \mathrm{L}$ prepared from a singleelement gold standard $(1000 \mu \mathrm{g} / \mathrm{mL}$; CGAUN1-125ML; Inorganic Ventures, Christiansburg, VA, USA). The acid blanks show a lower limit of detection (LOD; $3 \times$ standard deviation + mean) of $5.09 \mathrm{ng} / \mathrm{L}$ and a lower limit of quantification (LOQ; $10 \times$ standard deviation + mean) of $11.4 \mathrm{ng} / \mathrm{L}$.

\section{RESULTS AND DISCUSSION}

Gold nanoparticles are often surface-modified by a ligand exchange reaction using thiol containing organic compounds. For example, the use of 11-mercaptoundecanoic acid (MUA) results in a negatively charged surface. ${ }^{34}$ For use in nucleic acid delivery, cationic nanoparticles are employed for binding polyanionic nucleic acids, e.g., siRNA. Here, we employed thiol terminated LPEI to synthesize cationic gold nanoparticles (Figure 2A) for auropolyplex generation. This allowed direct ionic complexation of siRNA, thereby avoiding extensive purification steps and also enabling a simpler formulation approach.

Cationic Gold Nanocarrier for Auropolyplex Generation. Citrate stabilized gold nanoparticles were synthesized according to a protocol from Wurster et al., ${ }^{9}$ originally based on the report by Frens. ${ }^{30}$ Synthesis reproducibly resulted in monodisperse AuNPs with an average size of $68.5+/-2.8 \mathrm{~nm}$, as measured by NTA and visualized by TEM (Figure 2B,C). $\mathrm{UV}-$ vis spectroscopy of AuNPs demonstrated the presence of characteristic surface plasmon resonance (SPR) with an absorption wavelength maximum of around $530 \mathrm{~nm}$ (Figure S1), which matches with the reported data. ${ }^{9}$ Gold nanoparticles were directly surface-functionalized with thiol terminated LPEI (LPEI5) (Figure 2A). LPEI5 with a number average molecular weight of $5000 \mathrm{Da}$ was synthesized as hydrochloride salt by acidic hydrolysis of $\omega$-thiol terminated poly(2-ethyl-2-oxazoline) with $7 \mathrm{M} \mathrm{HCl} .{ }^{1} \mathrm{H}$ NMR analysis of LPEI5. $\mathrm{HCl}$ revealed complete cleavage of the side chain from $\alpha$-benzyl- $\omega$-thiol poly(2-ethyl-2-oxazoline), $\mathrm{CH}_{3}-\mathrm{CH}_{2}$-CO $[\delta$ $(\mathrm{ppm})=1.12-1.05]$, and $\mathrm{CH}_{3}-\mathrm{CH}_{2}-\mathrm{CO}[\delta(\mathrm{ppm})=2.45-$ 2.33], therefore showing full conversion of the precursor to thiol terminated LPEI (Figure S2). The thiol content of LPEI was evaluated by Ellman's assay, showing a thiol:LPEI ratio of 1:6. Surface functionalization of AuNPs with LPEI5 (thiol terminated LPEI) was carried out by the ligand exchange method and the successful formation of cationic AuNPs (here referred to as AL NP) characterized by UV-vis spectroscopy, NTA, and TEM. In addition to the thiol terminated LPEIS to AuNPs, the characteristic SPR band shifted to an absorption maximum of around $535 \mathrm{~nm}$ (Figure S1), indicating an increase of particle size and/or the chemisorption of thiol terminated LPEIS to the gold surface. Centrifugation-based purification allowed the removal of all unbound LPEIS and increased the concentration to an average of $2.32 \times 10^{11}+/-$ $6.28 \times 10^{9}$ nanoparticles/mL. Cationic AuNPs (AL) remained small $(71+/-2.8 \mathrm{~nm}$ by NTA), indicating the absence of any aggregation during the ligand exchange and purification step (Figure 2B). A change of the average $\zeta$-potential from $-27 \mathrm{mV}$ for citrate stabilized AuNPs to $+37 \mathrm{mV}$ for cationic AuNPs indicated successful assembly of thiol terminated LPEI5 on the surface of AuNPs (Figure 2B). More than 90\% of the gold nanoparticles were positively charged (Figure S3), indicating a homogeneous preparation of cationic AuNPs. TEM-based visualization indicated an average coating thickness of $6+/-$ $1.8 \mathrm{~nm}$ (29 fields of view analyzed) (Figure 2D). As there was only a minor size increase, we speculate that the LPEI layer rather appears in a "mushroom" state, i.e., not stretched like it would be in a "brush"-like structure. This is in accordance with the published literature: the hydrodynamic radius of nonaggregated LPEI $\left(M_{\mathrm{w}}=2500 \mathrm{Da}\right)$ has been reported to be 2.5 $\mathrm{nm}$, while the calculated contour length (stretched out) of a 5 $\mathrm{kDa}$ LPEI molecule is $\sim 38 \mathrm{~nm} .{ }^{35}$ In the case of PEG attached to AuNP, it has been observed that, with increasing PEG density, the hydrodynamic diameter increases and the brush structure occurs. ${ }^{36,37}$ Nevertheless, in our case, the coating density was appropriate to sufficiently stabilize the purified nanoparticles for at least 5 days at room temperature, indicating considerable stability. A positive $\zeta$-potential (Figure 2B) along with TEM visualization (Figure 2D) confirms the successful synthesis of cationic AuNP. Due to the postgrafting with thiol terminated LPEI5, cationic AuNPs remained homogeneous in size distribution. This is in contrast to a one-step synthesis process where thiolated PEI is already mixed with $\mathrm{HAuCl}_{4}$ during the nucleation process. ${ }^{38}$ The latter procedure rather leads to nanoparticles heterogeneous in size and shape.

Auropolyplexes: A Facile and Tunable siRNA-Nanotherapeutic Platform with High siRNA Loading Efficiency. Cationic AuNPs (AL NP) were used in a concentrated state to generate siRNA-based auropolyplexes by a simple twostep complexation process (as shown in Figure 1). The first step was to mix cationic AL NP with siRNA, giving siRNAloaded auropolyplexes ALS Aupx. In the second mixing step, the desired cationic polymer (LPEI5 (thiolated), LPEI10, or LPEI10-PEG; for details, see the Methods section) was added to ALS Aupx to ensure total complexation, giving respective auropolyplexes (ALSL5 Aupx; ALSL10 Aupx; ALSL10-PEG Aupx). The complexation process was characterized in detail by gel retardation studies, NTA, and TEM analysis. Gel retardation studies were conducted for different concentrations $(10 \mu \mathrm{g} / \mathrm{mL}$, relevant for in vitro applications; $133 \mu \mathrm{g} / \mathrm{mL}$, relevant for in vivo applications) and buffer conditions (water vs HBG; 20 mM HEPES pH 7.4, 5\% glucose w/v; Figure 3). When preparing ALS at $10 \mu \mathrm{g} / \mathrm{mL}$ in water, no retardation of siRNA was observed (complexation step 1; Figure 3A), but a black precipitate in the loading well could be seen visually and indicated the presence of gold nanoparticles in the well. In the second complexation step, increasing amounts of LPEI5 (from 10 to $400 \mathrm{ng}$ ) were added to ALS forming ALSL5 Aupx (complexation step 2; Figure $3 \mathrm{~A})$. At low $\mathrm{N} / \mathrm{P}$ ratios $(\mathrm{N} / \mathrm{P}$ $0.4-1$, corresponding to $10-25 \mathrm{ng}$ of LPEI5 per $200 \mathrm{ng}$ of siRNA), the intensity of the siRNA band gradually decreased, indicating partial retardation. At N/P 2 (50 ng of LPEI5) and above, a complete siRNA retardation occurred. Similarly, with free (untethered) LPEI5, siRNA was also completely retarded at N/P 2 and above (Figure S4). It is to be noted that, for the $\mathrm{N} / \mathrm{P}$ ratio calculation, the thiol terminated LPEI5 bound to AuNP was not considered.

Similar studies were conducted with a formulation intended for later in vivo use using AF750-labeled siRNA in HBG buffer at a siRNA concentration of $133 \mu \mathrm{g} / \mathrm{mL}$ and LPEI10 for complexation (Figure 3B). Almost complete retardation was 
A
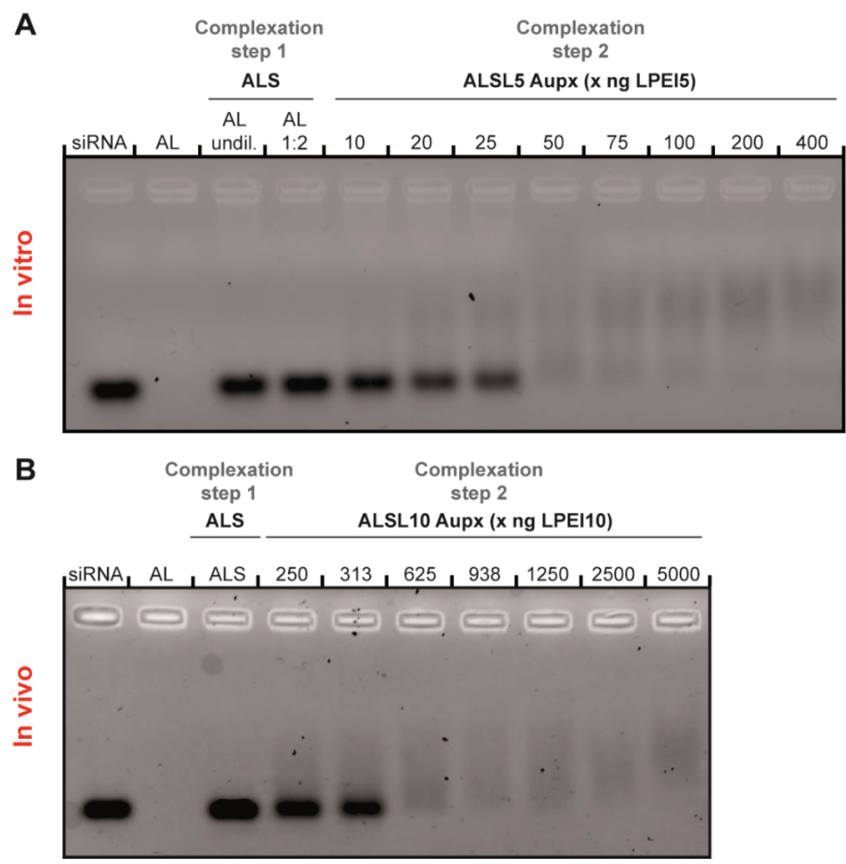

Figure 3. Gel retardation assay for studying siRNA complexation within auropolyplexes at siRNA concentration relevant for (A) in vitro and (B) in vivo studies. siRNA alone, AL NP alone, ALS (formed after complexation step 1, i.e., complexation of AL with siRNA), or ALSL auropolyplexes (formed after complexation step 2, i.e., complexation of ALS with indicated amounts of LPEIS or LPEI10 to form (A) ALSL5 or (B) ALSL10 Aupxs, respectively) were loaded onto a $1.5 \%$ agarose gel (200 ng of siRNA/lane; EtBr-stained) and underwent electrophoresis and visualization, as described in the Materials and Methods section. (A) Samples generated at a final concentration of 10 $\mu \mathrm{g} / \mathrm{mL}$ anti-luciferase siRNA, relevant for in vitro studies (in water). ALS generated with undiluted (undil.) or 1:2 diluted (1:2) AL; ALS (containing $200 \mathrm{ng}$ of siRNA in water) were condensed with indicated amounts of LPEI5, giving ALSL5 Aupx. (B) Samples generated at a final concentration of $133 \mu \mathrm{g} / \mathrm{mL}$ AF750-siRNA, relevant for in vivo studies (in HBG buffer). ALS Aupxs (containing $2500 \mathrm{ng}$ of siRNA) were condensed with indicated amounts of LPEI10, giving ALSL10 Aupx. For all samples, aliquots containing $200 \mathrm{ng}$ of AF750-siRNA each were loaded onto the gel.

observed at N/P of 2 and above (625 ng of LPEI per $2500 \mathrm{ng}$ of siRNA, complexation step 2; Figure 3B). Thus, gel retardations studies of auropolyplexes confirmed the total siRNA loading at N/P 2 and above. Sufficient surplus of positive charge ensures nanoparticle stability and transfection efficiency. ${ }^{17}$ Hence, for all further studies, we used formulations with higher N/P ratios: N/P 4 (corresponding to a $\mathrm{w} / \mathrm{w}$ ratio of $0.5 / 1 \mathrm{PEI} / \mathrm{siRNA}$ ) for in vitro studies and N/ P 8 (corresponding to a $1 / 1 \mathrm{w} / \mathrm{w}$ ratio of siRNA/LPEI) for in vivo studies.

We also applied $\zeta$-potential measurements to further characterize the individual complexation steps for different concentrations $(10 \mu \mathrm{g} / \mathrm{mL}$, relevant for in vitro applications; $133 \mu \mathrm{g} / \mathrm{mL}$, relevant for in vivo applications; Figure 4A). In the first complexation step, i.e., ALS Aupx synthesis at a concentration of $10 \mu \mathrm{g} / \mathrm{mL}$, the addition of siRNA to $\mathrm{AL}$ $\mathrm{NP}$ decreased the $\zeta$-potential from +37 to $-18 \mathrm{mV}$, indicating successful attachment/loading of negatively charged siRNA to the AL surface within ALS Aupx (Figure 4A). Apparently, the electrostatic interaction of the cationic AuNP surface with siRNA is sufficient to bind siRNA to AuNPs while they are in solution. However, this interaction appears to be not strong enough under conditions of gel retardation (high voltage) as siRNA was observed to be nonretarded for same parameters (Figure 3). This could be due to limited accessibility of thiol terminated LPEI5 tethered to the AuNP surface and/or low density of thiol terminated LPEI5 on the AuNP. Speculatively, this might be a reason for the probable presence of an unbound siRNA fraction but is unlikely to happen as complexation steps for formulating auropolyplexes are not done under high voltage conditions. However, owing to this observation, it is important to subject ALS Aupx to TEMbased visualization and was done in further experiments, as stated below. In the second complexation step, the addition of LPEI5 to ALS Aupx increased the $\zeta$-potential to $+24 \mathrm{mV}$ (Figure 4A), indicating complexation of siRNA into positively charged auropolyplexes and appears in line with the results obtained from the gel retardation assay. The second complexation step is critically needed to ensure the formation of cationic nanocarriers with excess LPEI on the surface. This excess LPEI plays an important role in nucleic acid delivery by enhancing cell uptake and endosomal escape, as observed in other studies. 39,40

In the case of auropolyplexes for in vivo setup $(133 \mu \mathrm{g} / \mathrm{mL}$ AF750 siRNA, LPEI10), $\zeta$-potentials of -26 (for ALS Aupx) and $+31 \mathrm{mV}$ (for ALSL Aupx) were recorded showing similar complexation behaviors (Figure 4A). Considering that the complexation was without additional purification steps, there is in principle the possibility of polyplex formation (in addition to or instead of auropolyplexes). To investigate this, we visualized the auropolyplexes by TEM (Figure S5A,B) to observe if only auropolyplexes are present or "polyplex-like structures", devoid of gold nanoparticles, are also present in addition to Aupxs. To properly identify polyplex-like structures, we also separately imaged polymer control treatment (i.e., without gold nanoparticles) of LPEI5 polymer + siRNA control (S5C, only the LPEI5 polymer mixed with siRNA) by TEM. In the case of auropolyplexes (Figure $\mathrm{S} 5 \mathrm{~A}, \mathrm{~B})$, only nanoparticles with high contrast and size range similar to AuNPs were observed for both ALS and ALSL Aupxs (four fields of view analyzed at a magnification of $200,000)$. On the other hand, the LPEI5 polymer + siRNA control appeared to be of low contrast with a bigger size of around $100 \mathrm{~nm}$ (Figure S5C) and exhibited a very low concentration of polyplex-like structures as only few particles were observed. Since such a low contrast and bigger structures were not observed in the case of both ALS and ALSL Aupxs, it indicates the absence of any detectable polyplexes during the process of auropolyplex generation. However, polyplexes might not be visible in the presence of gold nanoparticles because of their lower contrast in comparison to gold. To rule out this possibility and test if polyplex-like structures are visible in the presence of gold nanoparticles, we did a control experiment by deliberately mixing gold nanoparticles with precomplexed polymer + siRNA control and observed the mixture in TEM. Figure S6 shows that it is possible to visualize and discriminate polyplex-like structures from gold nanoparticles when they are present within a mixture. Also, as discussed above, it can be observed that polyplex-like structures are bigger in size in comparison to gold nanoparticles. Based on this observation, this scenario of lack of visibility of low-contrast polyplexes in the presence of high-contrast gold NPs can be ruled out in the case of Aupxs. Thus, there were no detectable polyplexes after the second complexation step in Aupxs, although the total 
Mean zeta potential

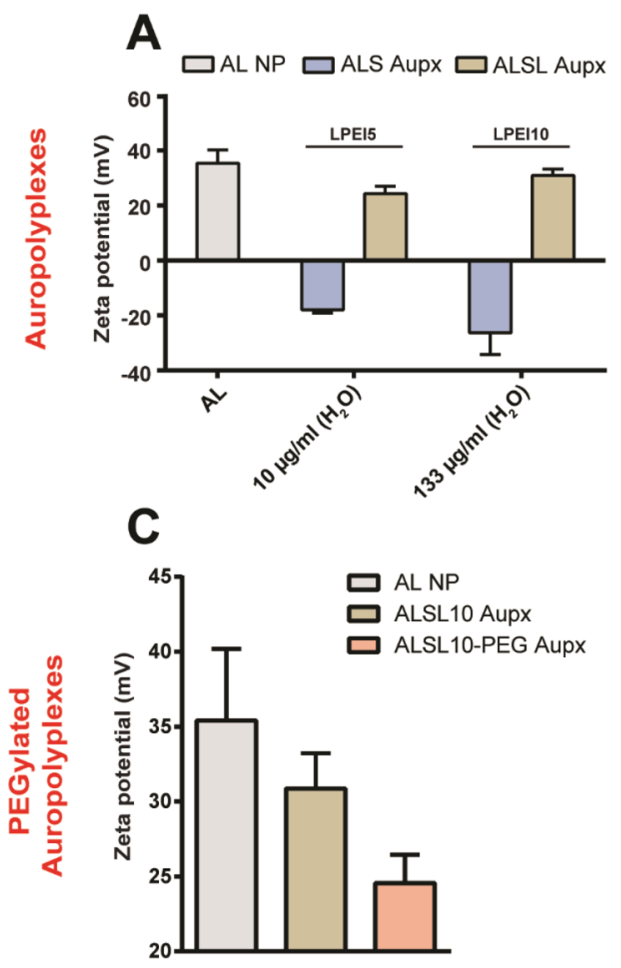

Mean particle size

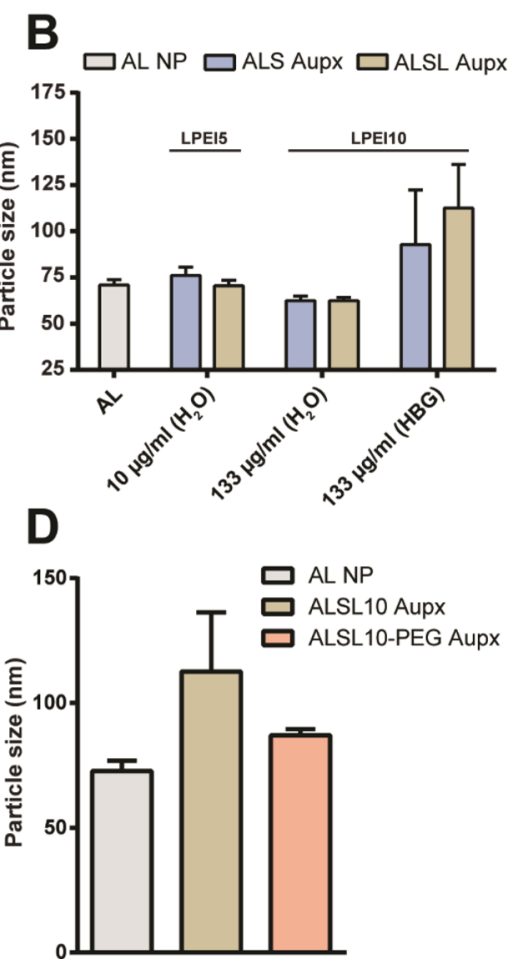

Figure 4. Nanoparticle tracking analysis of different steps of (A, B) auropolyplex generation and (C, D) PEGylation of auropolyplexes. Auropolyplex generation was characterized by NTA to give the (A) $\zeta$-potential and (B) mean size of nanoparticles at different steps (AL NP, cationic gold nanoparticles after chemisorption; ALS Aupx, after complexation of siRNA with AL NP, i.e., complexation step 1; ALSL Aupx, after complexation of ALS Aupx with LPEI5 or LPEI10 to form ALSL5 or ALSL10 Aupxs, respectively, i.e., complexation step 2). ALS and ALSL Aupxs were prepared in the indicated buffer (water or HBG); ALSL Aupxs were generated at a final siRNA concentration of $10 \mathrm{or} 133 \mu \mathrm{g} / \mathrm{mL}$ (as indicated), relevant for in vitro or in vivo studies, respectively. PEGylation of auropolyplexes was studied by NTA to characterize the (C) $\zeta$-potential and (D) mean particle size for PEGylated auropolyplexes (ALSL10-PEG Aupx) and compared with corresponding non-PEGylated auropolyplexes (ALSL10 Aupx) and cationic gold nanoparticles (AL NP); ALSL10-PEG and ALSL10 auropolyplexes were prepared in HBG buffer at a final siRNA concentration of $133 \mu \mathrm{g} / \mathrm{mL} ; n=3+\mathrm{SD}$.

absence of polyplexes or polyplex-like structures cannot be ruled out. Notably, the absence of polyplex-like structures within auropolyplexes is also supported by the observation of a highly negative $\zeta$-potential of ALS (after first complexation step) for both in vitro and in vivo setups (Figure 4A), indicating that siRNA is bound to $\mathrm{AL}$ and not available for complexation in the second step, thus hindering the possibility of polyplex formation (in addition to or instead of Aupxs).

Size-based characterization of auropolyplex generation for different concentrations $(10 \mu \mathrm{g} / \mathrm{mL}$, relevant for in vitro applications; $133 \mu \mathrm{g} / \mathrm{mL}$, relevant for in vivo applications) was conducted by NTA analysis (Figure 4B). All formulations generated in water, AL NP, ALS, and ALSL Aupxs exhibited average particle sizes between 60 and $75 \mathrm{~nm}$ (Figure 4B) in the case of both LPEI5 and LPEI10 auropolyplexes. Using HBG as the synthesis medium (relevant for in vivo application), ALS and ALSL10 auropolyplexes were $>100 \mathrm{~nm}$ in size. To determine whether this size increase in the case of ALSL10 Aupx is due to aggregation or due to an increased hydrodynamic diameter, NTA tracks of single nanoparticles in a defined volume were considered to give concentration of nanoparticles in water (Figure S7) and compared with physiological buffer HBG (Figure S8). Such an investigation is also relevant for studying the stability of ALSL10 auropolyplexes in physiologically relevant buffers like HBG. This can provide valuable information on size distribution data along with concentration of nanoparticles, which can be a potentially reliable indicator of stability of formulations. When ALSL10 Aupxs are subjected to NTA analysis in water (Figure S7), it seems that the size distribution and concentration profile did not change over multiple measurements for the same sample over a duration of 5-6 min, indicating good stability in water (for the tested duration). However, when the same sample of ALSL10 Aupxs was complexed and analyzed in HBG over the same duration, the size distribution and concentration profiles did change slightly with indications of a slight decrease or change in concentration and a very slight increase in size distribution over time, as can be observed in Figure S8. This indicates a slight trend toward aggregation for ALSL10 Aupx in HBG. The total particle count for ALSL10 Aupx generated in water was $2.98 \times 10^{11}+/-5.00 \times 10^{9}$ nanoparticles $/ \mathrm{mL}$, while in HBG buffer, it was $2.18 \times 10^{11}$ $+/-1.32 \times 10^{10}$ nanoparticles/mL (Figure S9). This indicates that not only the hydrodynamic diameter increased but also a minor fraction of nanoparticles was prone to aggregation when using $20 \mathrm{mM}$ HEPES and $5 \%$ glucose as a complexation medium. However, importantly, the fraction of particles below $200 \mathrm{~nm}$ in size for ALSL10 Aupxs generated in HBG was similar to that of Aupxs complexed in water (Figure S10). This indicates the absence of any drastic effect of HBG on auropolyplex nanoparticle population characteristics for the fraction of particles with a size of up to $200 \mathrm{~nm}$, which are 
A

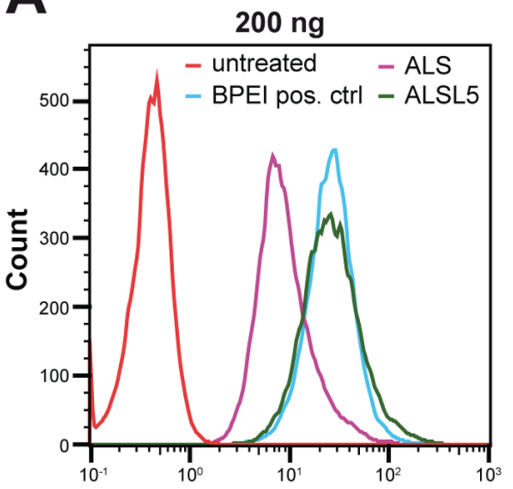

R1-A: AlexaFluor 647
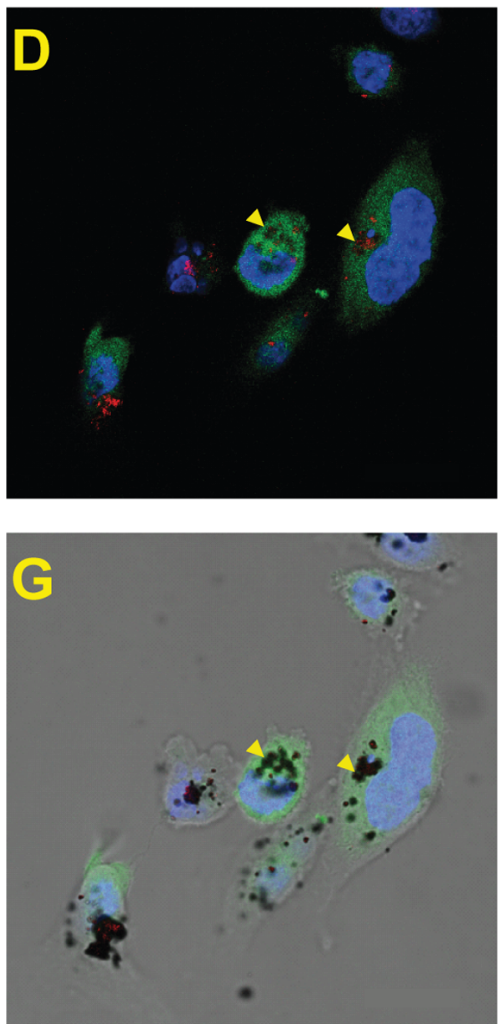

B

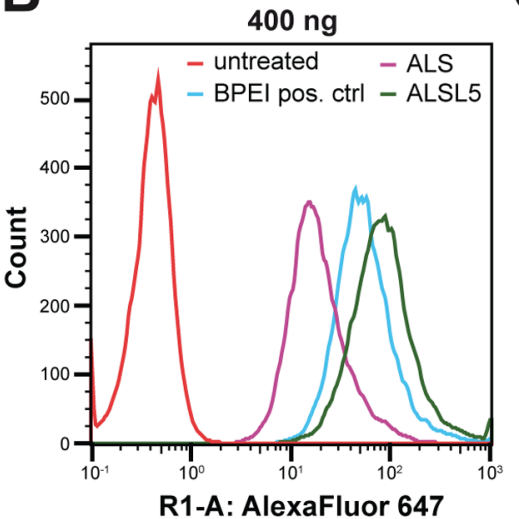

R1-A: AlexaFluor 647
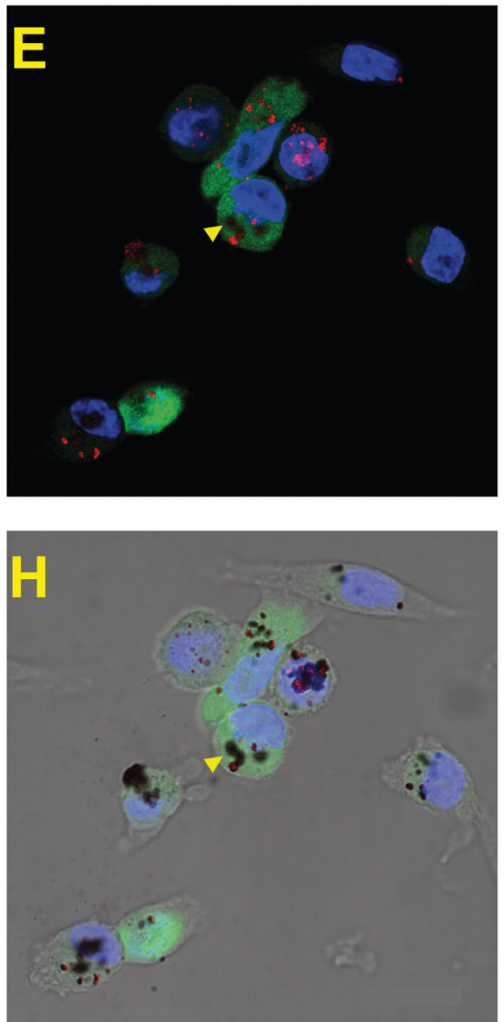

C
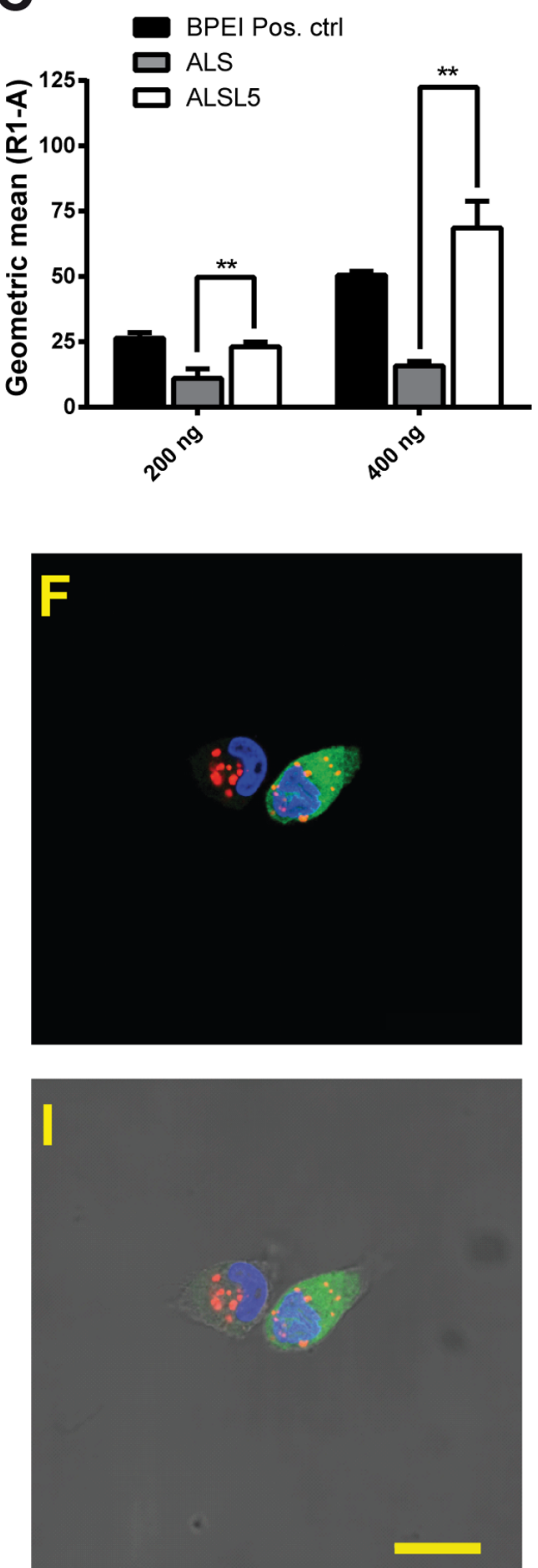

Figure 5. Cell association and uptake of auropolyplexes. MDA-MB-231Luc cells expressing the EGFP-luciferase fusion protein were incubated with AF647-siRNA-based ALSL (ALSL5) or ALS auropolyplexes (ALS) or BPEI-based polyplexes (as positive control, BPEI Pos. ctrl) for $24 \mathrm{~h}$ and analyzed for cell association by flow cytometry in comparison to $(A-C)$ untreated cells (untreated) or (D-I) cell internalization by confocal laser scanning microscopy. (A, B) Representative histograms for the AF647 signal in the R1 channel at a siRNA dose of (A) 200 or (B) $400 \mathrm{ng} /$ well. (C) Geometric mean values for the AF647 signal in the R1 channel for different treatments $(n=3+\mathrm{SD}$, data from two independent experiments; $* * p$ $\leq$ 0.01; U-test (Mann-Whitney). (D-I) CLSM-based imaging of the middle section of the cells showing (D-F) fluorescence images or (G-I) DIC overlay with fluorescence images after incubation with (D, G) ALS Aupx, (E, H) ALSL5 Aupx, or (F, I) BPEI polyplexes. DAPI staining is depicted in blue, the EGFP signal (expressed by cells constitutively) in green, and the AF647 signal derived from siRNA in red; arrowheads in panels (D, E) denote areas without EGFP signal and in panels $(\mathrm{G}, \mathrm{H})$, the corresponding area in the figure with DIC overlay; scale bar, $20 \mu \mathrm{m}$.

important for transfection. ${ }^{41}$ To demonstrate the versatility of auropolyplex formulation and investigate the effect of PEG on stability, LPEI10-PEG was used instead of LPEI10 to prepare PEGylated auropolyplexes (Figure 4C,D) at $133 \mu \mathrm{g} / \mathrm{mL}$ with AF750-siRNA in HBG without involving any complicated PEGylation steps. Inclusion of stealth attributes within auropolyplexes in a facile manner is an advantage of the twostep complexation approach and is relevant from the standpoint of preparation of potentially upscalable in vivo formulations. The $\zeta$-potential was lower for PEGylated auropolyplexes (Figure 4C), and the average hydrodynamic diameter of PEGylated auropolyplexes was around $87 \mathrm{~nm}$ (Figure 4D) and smaller in comparison to their nonPEGylated version in HBG buffer. This is also supported by the relatively stable size and concentration distribution profile (over multiple measurements) for PEGylated ALSL10 auropolyplexes in HBG (Figure S11) in comparison to nonPEGylated ALSL10 auropolyplexes in HBG (Figure S8). This masking of surface charge and prevention of aggregation on inclusion of PEG is in accordance with the effects of PEGylation on gold and other nanoparticles. ${ }^{36,42}$ Also, the fraction of particles below $200 \mathrm{~nm}$ in size for PEGylated 
ALSL10 Aupxs in HBG is similar to that of ALSL10 Aupxs complexed in water (Figure S10). Thus, PEGylated and nonPEGylated ALSL10 Aupxs were relatively stable in HBG buffer (for the tested duration), which is relevant for their potential in vivo applications.

The amount of siRNA loaded per nanoparticle can be crucial for an effective knockdown and prolonged biological effect (especially in vivo) because of inefficient cell uptake and low rates of endosomal escape. ${ }^{3,4}$ This is further compounded by toxicity associated with high nanoparticle loads to deliver the required efficacious dosage. We estimated $\sim 15,500$ siRNA duplex molecules per auropolyplex particle (in the case of ALSL10 generated with AF750-siRNA at $133 \mu \mathrm{g} / \mathrm{mL}$ in water, considering complete association of siRNA, as confirmed by gel retardation data; see details for calculation in the Methods section). This is considerably higher when compared to LBL techniques (3500 duplex molecules per particle ${ }^{4}$ ) or with covalently attached siRNA, which was $\sim 38$ duplexes for a 13 nm particle. ${ }^{43,44}$ Thus, optimized auropolyplexes exhibit a high and tunable loading efficiency $(10 \mu \mathrm{g} / \mathrm{mL}$, relevant for in vitro applications; $133 \mu \mathrm{g} / \mathrm{mL}$, relevant for in vivo applications), which is a key attribute needed for efficient in vivo performance by nucleic acid nanotherapeutics.

Auropolyplexes for Successful Intracellular siRNA Delivery and Gene Knockdown. Cell binding, internalization, and endosomal release are key steps in siRNA delivery. Here, we employed net positively charged nanoparticles, which usually bind via heparansulfate proteoglycans and are internalized by adsorptive endocytosis. ${ }^{45}$ AF647-labeled siRNA-based auropolyplexes were employed to detect their binding and uptake by flow cytometry (Figure 5A,C) and CLSM (Figure 5D-I). Cell uptake experiments of all formulations were conducted in the presence of serum for $20 \mathrm{~h}$ after an initial $4 \mathrm{~h}$ incubation step of formulations with cells in a basal medium. Cell association of AF647-siRNAbased nanoparticles significantly increased when the dose was doubled from 200 (64 nM siRNA) to $400 \mathrm{ng} /$ well (128 nM siRNA), as can be seen in Figure 5A-C. ALSL auropolyplexes showed high cell association, whereas negatively charged ALS Aupx exhibited significantly lower total cell association (Figure 5C). This indicates the need of a cationic surface charge for high cellular association, which translates into a high cell uptake. Notably, the cationic charge on ALSL Aupx is ensured by the second complexation step to include excess LPEI. BPEI polyplexes were used as an internal positive control and also showed cell association higher than ALS Aupx but comparable to ALSL5 Aupx. LPEI5 polymer + siRNA control (L5 polymer control) showed similar uptake as corresponding ALSL5 auropolyplexes (Figure S12A). Confocal laser scanning microscopy (CLSM) was used to validate uptake and visualize intracellular distribution at a concentration of $128 \mathrm{nM}$ siRNA (Figure 5D-I; Z-stacks as video files in the Supporting Information). The fluorescent signal of EGFP (from the EGFP-Luc fusion protein produced by cells under constitutive expression) was utilized to visualize the cytoplasmic area and thereby investigate the localization of AF647-siRNA in this spatial context. As observed in the middle sections of cells (within the Z-stack), AF647-labeled siRNA was found intracellularly in all treatments, ALS (Figure 5D and Video S1), ALSL auropolyplexes (Figure 5E, Videos S2 and S3), and BPEI-positive control (Figure 5F and Video S4). In transmitted light DIC pictures (overlaid onto fluorescence micrographs), dense structures (appearing black) were visible within
ALS and ALSL5 auropolyplex-treated cells but not with BPEI polyplex transfected ones (Figure 5G-I). Comparing DIC and CLSM pictures, there is an intriguing correlation between the dark structures in DIC and the absence of the EGFP signal in the corresponding areas in the CLSM picture (highlighted by arrows), which indicates the presence of gold nanoparticles intracellularly. These structures also resembled the same distribution pattern as the AF647 signal, indicating the presence of siRNA intracellularly near to gold NPs. In the case of ALS Aupx, although the total cellular association of siRNA was significantly lower (5C), internalization was still observed (5D and 5G). Patel et al. reported a similar observation where a negatively charged AuNP covalently decorated with siRNA was internalized after binding to scavenger receptors. ${ }^{46}$ Taken together, we conclude that cationic ALSL auropolyplexes and siRNA are efficiently delivered intracellularly. Further, we also investigated in vitro biocompatibility behavior of these formulations as cationic polymers are known to be toxic to cells. ${ }^{16}$ Figure S13 shows the percentage cell viability of auropolyplexes and is in the range of $60-80 \%$.

Several uptake routes for siRNA and formulations thereof have been described, but only a few lead to an efficient target knockdown. ${ }^{47}$ After establishing the successful intracellular siRNA delivery, we therefore assessed gene knockdown efficiency of auropolyplexes on MDA-MB-231Luc cells stably expressing the EGFP-Luc reporter gene. Cells were treated with different formulations based on anti-luc siRNA or noncoding scrambled control siRNA (400 ng/well, corresponding to $140 \mathrm{nM}$ ) for $48 \mathrm{~h}$, and the luciferase activity was quantified by the firefly luciferase assay. The luciferase signal from cells treated with anti-luc siRNA-based formulations was normalized to cells treated with formulations loaded with noncoding scrambled control siRNA (Figure 6). Gene knockdown experiments of all formulations were conducted

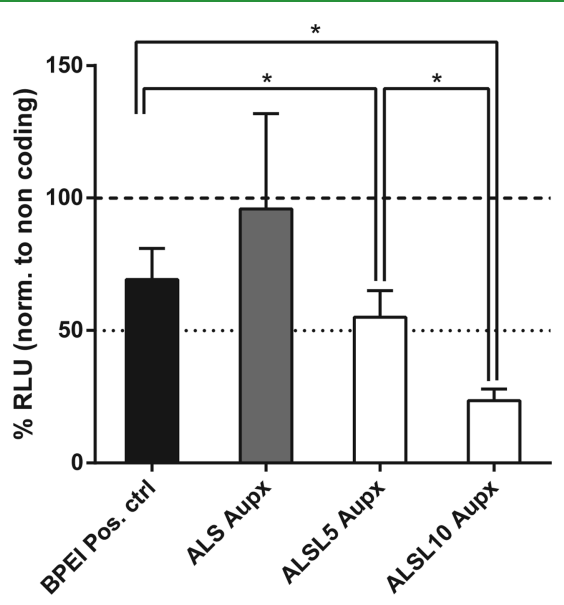

Figure 6. Functional evaluation of siRNA-based gene knockdown by auropolyplexes. MDA-MB-231Luc cells expressing the luciferasebased reporter gene under a constitutively active promoter were treated with anti-luc-siRNA or noncoding scrambled control siRNA (400 ng of siRNA/well; $140 \mathrm{nM}$ ) formulated as ALSL auropolyplexes (ALSL5 or ALSL10 Aupx) or ALS auropolyplexes (ALS Aupx) or BPEI-based polyplexes (as positive control, BPEI Pos. ctrl) for $48 \mathrm{~h}$ and assayed for luciferase expression. Relative knockdown in percent (\% RLU) is calculated by the formula $100 \times$ (RLU anti-luc siRNA/ RLU noncoding scrambled control siRNA); $n=3+\mathrm{SD}$, data from two independent experiments; ${ }^{*} p \leq 0.05$; $U$-test (Mann-Whitney). 

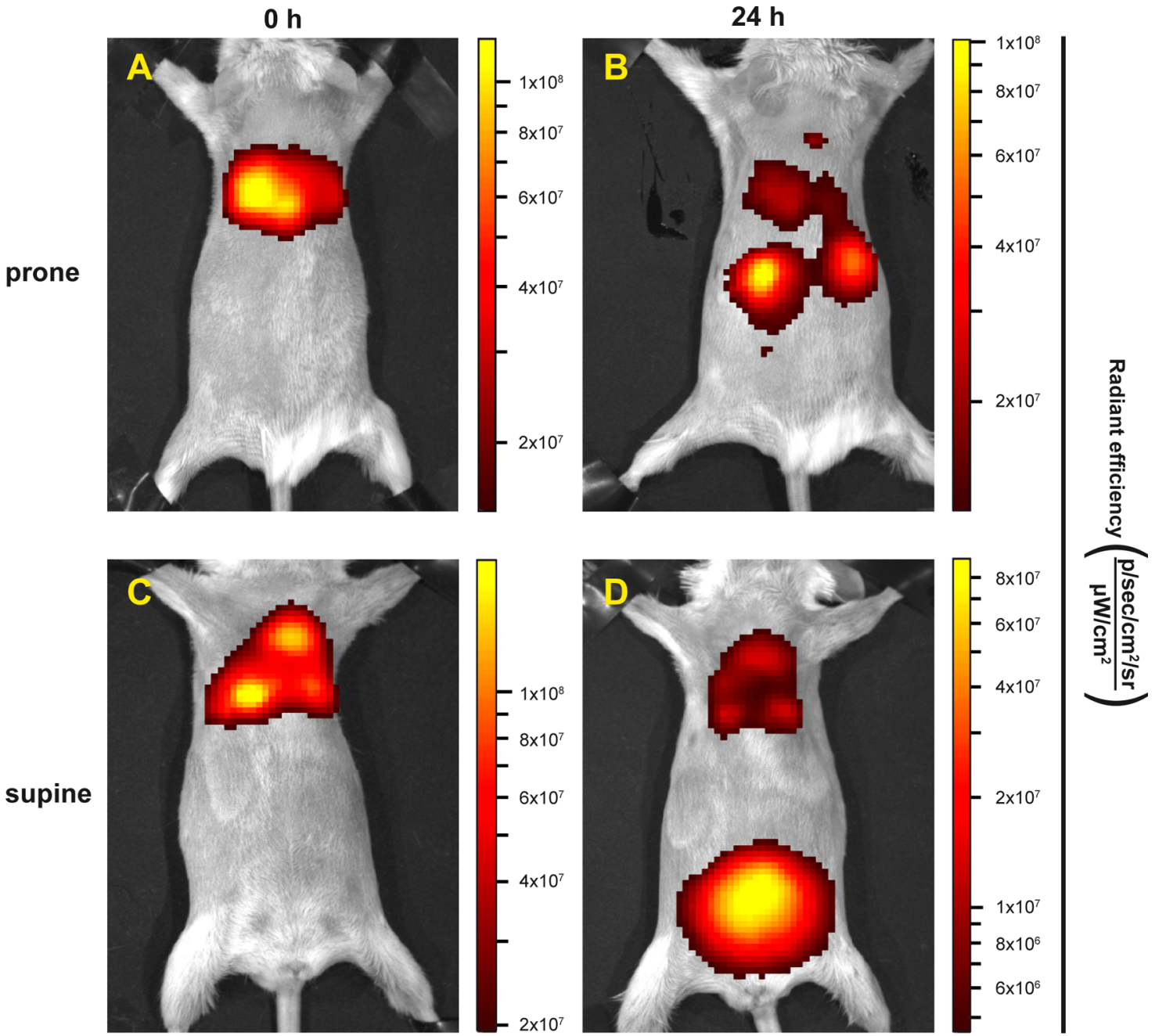

Figure 7. 2D epifluorescence imaging-based biodistribution of AF750-siRNA after intratracheal pulmonary delivery of auropolyplexes. Balb/c mice were treated with ALSL10 auropolyplexes (containing $10 \mu \mathrm{g}$ of AF750-siRNA) intratracheally by microspray-based aerosolization and imaged immediately after $(\mathrm{A}, \mathrm{C})$ application $(0 \mathrm{~h})$ and $(\mathrm{B}, \mathrm{D}) 24 \mathrm{~h}$ thereafter either in a $(\mathrm{A}, \mathrm{B})$ dorsal (prone) or $(\mathrm{C}, \mathrm{D})$ ventral up (supine) position for AF750 fluorescence signal. Color-coded fluorescence radiance images are overlaid onto reflected light images; $n=3$ per group, representative animals are shown.

in the presence of serum for $44 \mathrm{~h}$ after an initial $4 \mathrm{~h}$ incubation step of formulations with cells in the basal medium. Both ALSL5 and ALSL10 auropolyplexes caused a significantly reduced luciferase expression, demonstrating efficacious delivery of siRNA in the case of both auropolyplexes. In contrast, ALS Aupx did not show any reduction in luciferase expression, which might be ascribed to their low cell uptake, as observed from the flow cytometry data (Figure 5C). Although ALS Aupxs were internalized (Figure 5D,G), their lack of knockdown efficiency points to insufficient siRNA delivered intracellularly, as observed in their significantly low geometric mean in comparison to ALSL5 auropolyplexes (Figure 5C). Additionally, the presence of excess LPEI in ALSL Aupx (in comparison to ALS Aupx) also ensures adequate endosomal escape, which is crucial for successful siRNA delivery, as also observed in other studies. ${ }^{39,40}$ Notably, it has been observed that, for in vitro activity of nucleic acid polyplexes, a minimum amount of polyethylenimine is needed to achieve biological activity of delivered nucleic acid, which is in part due to the content of free polyethylenimine, which is cointernalized and aids in endosomal escape. ${ }^{39,48}$ Furthermore, in this case, the total amount of biologically active nucleic acid is not that critical and can also be partially replaced by biologically inactive "stuffer" polyanions, as reported by Kichler et al. ${ }^{49}$ BPEI $\left(M_{\mathrm{w}}=25,000 \mathrm{Da}\right)$ polyplexes also showed knockdown in luciferase expression and were used as an internal positive control based on their gene silencing potential, as reported in other studies. $^{16,50}$ When comparing ALSL5 and ALSL10 auropolyplexes with their corresponding polymer + siRNA control (i.e., without gold nanoparticles; L5 polymer + siRNA control or L10 polymer + siRNA control, respectively), the knockdown was either slightly improved (for ALSL10 Aupx) or similar (for ALSL5 Aupx; Figure S12B). The knockdown of ALSL10 Aupx in comparison to the L10 polymer control indicates the probable role of gold nanoparticles in assisting siRNA delivery and knockdown. A similar assisting role of nanoparticles in gene delivery was described for BPEI-based plasmid delivery. ${ }^{38}$ Surprisingly, gene knockdown performance of L5 polymer + siRNA control (L5 polymer ctrl; Figure $\mathrm{S} 12 \mathrm{~B})$ can be ascribed, speculatively, to the presence of terminal thiol in the case of the L5 polymer, i.e., thiol terminated LPEI5 (not present in the case of the L10 polymer, i.e., LPEI10). However, this needs further investigation both at a physico-chemical level and biological characterization. 

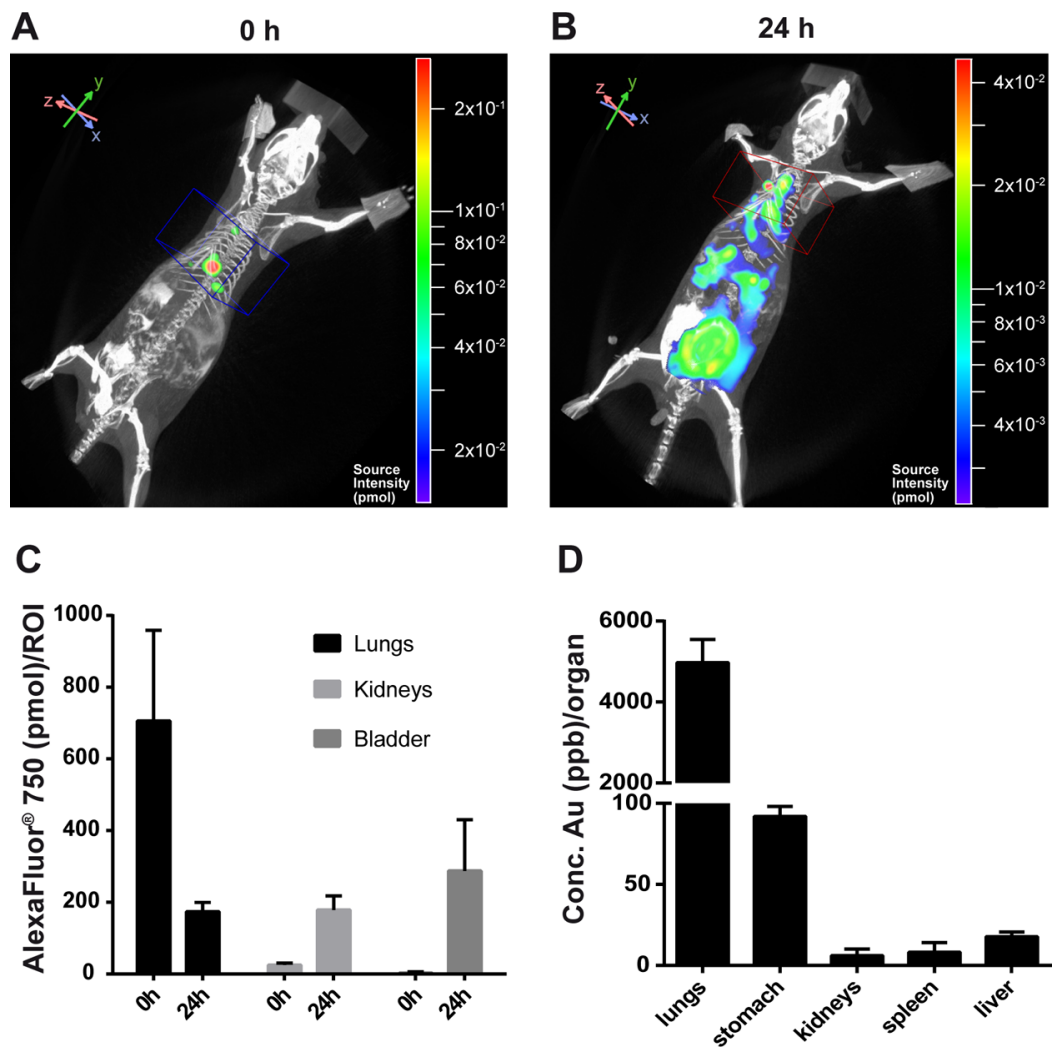

Figure 8. Fluorescence imaging tomography/X-ray absorption computed tomography (FLIT/CT)-based spatio-temporal and noninvasive tracking of the (A, B) siRNA delivery process followed by (C) AF750-siRNA quantification in vivo (by FLIT/CT based absolute quantification) and (D) gold quantification from organs ex vivo (by inductively coupled plasma mass spectrometry, ICP-MS). Balb/c mice were intratracheally treated with ALSL10 auropolyplexes loaded with $10 \mu \mathrm{g}$ of Alexa750-siRNA, as described. (A, B) FLIT/CT-based tracking of AF750-siRNA biodistribution within the animal noninvasively and at different time points $(0$ and $24 \mathrm{~h}$ after intratracheal application); fluorescence signal color coded in pmol units and CT in black/white. (C) Quantification of the AF750-siRNA signal within the animal by FLIT image analysis in the lung region, kidney area, and bladder area at 0 and $24 \mathrm{~h}$ after intratracheal administration. (D) ICP-MS-based quantification of gold per organ $24 \mathrm{~h}$ after intratracheal application; $n=3+\mathrm{SD}$.

ALSL10 auropolyplexes performed superiorly with an $80 \%$ knockdown and were thus used for further in vivo biodistribution studies. Very recently, SNAs (siRNA attached to $13 \mathrm{~nm}$ AuNP) were complexed with $25 \mathrm{kDa}$ BPEI and used for in vitro transfection studies. ${ }^{44}$ Based on siRNA, $60 \mathrm{nM}$ were necessary to achieve a $70 \%$ reporter gene (EGFP) knockdown at a concentration of $34 \mu \mathrm{g} / \mathrm{mL}$ of BPEI (giving an $\mathrm{N} / \mathrm{P}$ ratio of $\sim 630$ and a siRNA/BPEI $w / w$ ratio of $41 / 1)$. Although the formulation was very well tolerated in vitro, BPEI dosages of 2 $\mathrm{mg} / \mathrm{kg}$ and above induce acute toxicity with $\sim 50 \%$ lethality, ${ }^{17}$ hence limiting the treatment dosage of siRNA in vivo with BPEI-based formulations. Considering the toxicity profile of BPEI and better biocompatibility of LPEI-based formulations, ALSL10 auropolyplexes are promising and were thus employed for studying siRNA delivery and release in vivo.

As a proof of principle, we also evaluated PEGylated auropolyplexes (ALSL10-PEG Aupx) generated with LPEI10PEG for cell uptake and gene knockdown at 70 and $140 \mathrm{nM}$ (Figure S14). Despite the presence of PEG, ALSL10-PEG auropolyplexes showed similar cell association/uptake as in the case of ALSL10 auropolyplexes (Figure S14A). As expected, knockdown by PEGylated auropolyplexes was less pronounced in comparison to non-PEGylated auropolyplex (ALSL10 Aupx) but still showed silencing in gene expression (Figure S14B). Thus, PEGylated auropolyplexes are potentially suitable for targeted siRNA delivery, e.g., to EGFR overexpressing cells, similar to our work on targeted gene delivery by LPEI10-PEG-based conjugates with distally attached peptide ligands. $^{51,52}$ Thus, the auropolyplex platform can be in principle used for developing targeted and shielded nucleic acid delivery formulations.

Pulmonary siRNA Delivery by Aerosolization of Auropolyplexes: FLIT/CT-Based Spatio-Temporal and Noninvasive Tracking of the siRNA Delivery Process. Pulmonary delivery of nucleic acids is very relevant for the treatment of lung-related disorders as it offers local distribution and higher lung retention, thereby circumventing accumulation in nontarget organs and avoiding side effects. Delivery by microspray-based aerosolization via an intratracheal administration route is generally employed for proof-of-concept animal studies. ${ }^{53,54}$ We have recently applied naked but chemically modified siRNA intratracheally demonstrating not only partial lung retention but also crossing of the air-blood barrier and renal excretion of intact siRNA. ${ }^{24}$ Also, we could confirm that the AF750 dye remains attached to siRNA and the siRNA remains intact when analyzed in urine, making this a suitable method for tracking siRNA in vivo. Notably, the presence of intact siRNA in urine could be due to the employment of chemically modified siRNA, which showed better in vivo stability, which is crucial for its biological function. Considering this observation, we employed the same chemically modified siRNA to formulate auropolyplexes in the present work. Here, we evaluated the biodistribution and biocompatibility of ALSL10 auropolyplexes with AF750- 
siRNA in HBG $(133 \mu \mathrm{g} / \mathrm{mL})$ in vivo via the intratracheal administration route. After intratracheal application of ALSL10 auropolyplexes formulated with $10 \mu \mathrm{g}$ of AF750-siRNA, the $2 \mathrm{D}$ epifluorescence signal was found in the thoracic area within minutes, indicating deposition in the lung $(0 \mathrm{~h}$; Figure $7 \mathrm{~A}, \mathrm{C})$. After $24 \mathrm{~h}$ of administration, a strong signal was also observed in the abdominal area, depicting signal in kidneys (when imaged in the prone position; Figure $7 \mathrm{~B}$ ) and in the bladder area (when imaged in the supine position; Figure $7 \mathrm{D}$ ), indicating distribution from the lungs to excretory organs. To aid $2 \mathrm{D}$ epifluorescence imaging results, which lack exact organ allocation and absolute quantification, 3D fluorescence imaging (FLIT) in combination with X-ray computed tomography was performed. When applying tomographic analysis together with contrast agent-enhanced CT (iopamidol for improving abdominal organ delineation), the distribution of the fluorescence signal from the respiratory tract (blue ROI in Figure $8 \mathrm{~A}$ ) to the renal system $24 \mathrm{~h}$ after administration (Figure 8B) was confirmed corroborating the 2D analysis. Similarly, ex vivo imaging of organs corroborated the observation with a significant signal in both kidneys and in the lung (Figure S15).

Fluorescence imaging tomography/X-ray absorption computed tomography (FLIT/CT) imaging allowed noninvasive spatio-temporal tracking (Figure $8 \mathrm{~A}, \mathrm{~B}$ ) and quantitative analysis of fluorescence signal within the animal at 0 and 24 $\mathrm{h}$ after i.t. administration (Figure $8 \mathrm{C}$ ). Quantification was done by employing a standard curve based on different concentrations of AF750 siRNA, which was then used to quantify the fluorescence signal within appropriately placed cuboid $3 \mathrm{D}$ ROIs within FLIT images of the animal, as recently described. ${ }^{24,25}$ FLIT images of animals at 0 and $24 \mathrm{~h}$ after i.t. administration of auropolyplexes were analyzed for the absolute amount of fluorescence by placing 3D ROIs in the lung area, kidneys, and bladder area, as shown in Figure S16. Iopamidol-based CT contrast helped in delineation of organs like the kidneys and bladder (Figure S16). Absolute quantification of the AF750-siRNA signal (in pmol) was then plotted (Figure 8C) and shows a decrease in the lung area signal and an increase in the signal in the kidneys and bladder area $24 \mathrm{~h}$ after administration of Aupxs. Based on a $M_{\mathrm{w}}$ of $15,700 \mathrm{Da}, 10 \mu \mathrm{g}$ of AF750 siRNA corresponds to $637 \mathrm{pmol}$ AF750. Immediately after spraying $705+/-252$ pmol, AF750 were found in the lung area, indicating a complete lung delivery of the dosage applied (recovery rate $\sim 110 \%$ ). Twentyfour hours thereafter, $\sim 25 \%$ of the dosage $(173+/-26 \mathrm{pmol})$ was found in the lung, whereas the remaining material was apparently excreted renally, as can be observed in terms of an increase in the fluorescence signal in the kidneys and bladder (Figure $8 \mathrm{C}$ ), which is also corroborated by ex vivo organ imaging (Figure S15) performed just after FLIT/CT. Noninvasive estimation of the amount of AF750-siRNA delivered in vivo in target organs can significantly help in assessing the therapeutic potential of nanotherapeutics within preclinical and clinical studies. Overall, the results from 2D epifluorescence- and FLIT/CT-based whole-body imaging and ex vivo organ analysis indicate air-to-blood transfer and then renal excretion of AF750-siRNA after intratracheal administration of ALSL10 auropolyplexes (loaded with AF750-siRNA). This is in line with the other studies where siRNA is renally excreted after air-blood transfer, as studied by fluorescence, histology, or radioactive methods. ${ }^{50,55}$ Notably, this kind of noninvasive spatio-temporal tracking and absolute quantification of drug
(AF750-siRNA) dose delivered to the target organ(s) is a crucial step (within nanotherapeutic characterization) before going forward with in vivo functional studies where the biodistribution pattern and delivered dosage can be decisive for the overall therapeutic potential.

One important aspect of carrier-mediated siRNA delivery, which is very often not studied, is the investigation of biodistribution of the delivery vehicle per se once the drug is delivered in vivo. Knowledge of in vivo biodistribution of the drug carrier per se can give important insights regarding the delivery process, for example, if the drug (siRNA in this case) was released so as to be available for its biological function. Toward this goal, we also quantified the amount of gold remaining in the respective organs using ICP-MS (Figure $8 \mathrm{D}$ ) after sacrificing the animals. ICP-MS measurements showed that, $24 \mathrm{~h}$ after auropolyplex administration, $>97 \%$ of the detected gold remained in the lungs at a concentration of 4970 +/- $469 \mathrm{ppb}$. In the stomach, $1.8 \%$ of the dose was found, whereas in all other organs, values were below $0.5 \%$, which was in the range of acid blank measurements. The amount of gold in the stomach was very low and can be due to the activity of the mucociliar escalator. Comparable results were reported for noncationic $22 \mathrm{~nm}$ AuNP where values of $>3 \%$ stomach retention were observed already $2 \mathrm{~h}$ after intratracheal application. ${ }^{56}$ In the case of Aupxs, the biodistribution pattern of gold (via ICP-MS) is quite different from the AF750-siRNA biodistribution, as measured by fluorescence, indicating no apparent air-blood transfer of AuNP and strong retention of the gold in the lungs. This difference cannot be explained only due to the sheer differences in size: $2 \mathrm{~nm}$; citrate-capped AuNPs remained in the lung with only minute amounts found in the liver, as per Sadauskas et al. ${ }^{57}$ In contrast, siRNA of a similar size ( $2 \mathrm{~nm}$, when considering the hydrodynamic diameter $)^{58}$ crosses the air-blood barrier rapidly and efficiently and is excreted via kidneys. ${ }^{24,55}$ Thus, in the case of auropolyplexes, the release of siRNA from gold AuNP seems unhampered, which can be advantageous from the standpoint of functionality. In contrast, covalently attached nucleic acid to the gold NP surface is not completely released where $\sim 60 \%$ remains surface-bound ${ }^{13}$ and might not be accessible to the cellular machinery. The rationale of using thiol terminated LPEI (as in the case of auropolyplexes) rather than thiolatedsiRNA (as in the case of SNAs) was to avoid trapping of siRNA onto the gold nanoparticles after intracellular delivery, thereby ensuring the release of siRNA also inside the cells. Importantly, in case a fraction of siRNA is released from the auropolyplex before cell uptake, it can still avoid degradation and perform its biological function owing to the usage of chemically modified siRNA for auropolyplex formulation.

LPEI-based auropolyplexes were well tolerated in vivo for the tested duration and seem to offer a good balance between a successful endosomal escape (owing to high siRNA loading) and acceptable biocompatibility profile. After intratracheal administration of ALSL10 auropolyplexes, no immediate toxicity was observed in the treated animals. Sometimes, there was momentary coughing directly after instillation, which was associated with this administration route. The animals recovered well after the administration and imaging session. Gross pathological examination of organs showed no macroscopic differences between treated and untreated control animals. This can be attributed to the usage of the linear version of PEI rather than the branched format (which is associated with acute toxicity ${ }^{17}$ ). LPEI-based formulations are 
under several preclinical and clinical trials for a variety of applications like DNA vaccine ${ }^{21}$ and cancer therapy (bladder and pancreatic cancer treatments $)^{20,22,59,60}$ and are considered to be of potential interest for in vivo nucleic acid therapy. Considering the biocompatible nature of gold nanoparticles, LPEI-based auropolyplexes are promising nucleic acid nanocarriers for efficacious delivery in vivo. However, detailed nanotoxicological studies are needed to fully investigate their in vivo biocompatibility behavior. Although gold nanoparticles are retained in the lungs for longer duration, this does not affect their therapeutic potential as evident from similar studies with pulmonary delivery of gold nanoparticles. Sung et al. conducted a subchronic toxicity study on inhaled gold nanoparticles in rats and showed only minimal and statistically nonsignificant effects on lung function and inflammation, even upon chronic exposure (up to 90 days).$^{61}$ Functionality testing, i.e., target knockdown in vivo, and investigation of cellular uptake/localization of AF750 siRNA in vivo were not conducted with the current version of auropolyplexes as these studies are more relevant within a disease context where the targeted version of auropolyplexes can be used and is the next step. Also, for a healthy lung tissue, the mucus represents a strong biobarrier for nanoparticles usually hindering their access to lung cells. ${ }^{62}$ However, in principle, targeted and PEGylated auropolyplexes might be applicable in lung cancer models based on our recent results where we could demonstrate accessibility of CD49f-overexpressing breast cancer lung metastases for gene transfection by peptidetargeted polyplexes via intratracheal administration due to the invasive growth of cancer cells. ${ }^{63}$

\section{CONCLUSIONS}

Preclinical/clinical translation of nucleic acid nanomedicines is decisively dependent on critical formulation parameters, which should be considered already during the design and development phase. Toward this, combination of chemisorption (of cationic polymer onto gold NPs) and complexation (with siRNA) allows tailorability in auropolyplex formulation, thereby enabling tunable biophysical properties for overcoming multiple challenges relevant for efficient siRNA therapeutics. The robust synthesis protocol enables generation of auropolyplexes for in vivo delivery with sufficient siRNA concentration in physiological buffers and with precise control on loading efficiency. Tunability of siRNA loading is demonstrated by preparing auropolyplexes at siRNA concentrations of 10 and $133 \mu \mathrm{g} / \mathrm{mL}$ for in vitro and in vivo experiments, respectively. They offer effective siRNA delivery with exceptionally high loading capacity per NP, efficient cellular uptake, and intracellular release of siRNA, thereby exhibiting a good knockdown efficiency. Facile synthesis and a modular structure allow tunable loading of siRNA and the use of shielding polymers (PEGylated Aupxs) and also potentially targeting agents in an upscalable fashion for cell-specific siRNA delivery. Fluorescence and X-ray absorption-computed tomography allowed noninvasive spatio-temporal tracking of the siRNA delivery process and revealed successful pulmonary delivery, unhampered release of siRNA in vivo from the nanocarrier followed by air-blood barrier crossing, and excretion via the renal system. Importantly, noninvasive absolute quantification of the delivered dose of AF750siRNA in the lungs, kidneys, and bladder was demonstrated, which can significantly aid in preclinical assessment of nanotherapeutics. Considering the current clinical studies on linear polyethylenimine-based formulations, LPEI-based auropolyplexes offer a promising combination of successful siRNA delivery and better biocompatible profile in vivo. Taken together, auropolyplexes represent a versatile nanotherapeutic platform with crucial attributes for efficient siRNA delivery for potential preclinical studies and, in principle, also for other similarly sized nucleic acids.

\section{ASSOCIATED CONTENT}

\section{Supporting Information}

The Supporting Information is available free of charge at https://pubs.acs.org/doi/10.1021/acsami.0c06608.

UV-vis absorbance, ${ }^{1} \mathrm{H}$ NMR analysis, gel retardation data, TEM images, nanoparticle tracking analysis-based size and concentration distribution, in vitro cell association, gene knockdown and biocompatibility, ex vivo fluorescence imaging, and fluorescence imaging/Xray absorption computed tomography (PDF)

CLSM-based video file showing Z-stacks of EGFP-Luc expressing MDA-MB-231 treated with ALS auropolyplexes based on AF647-labeled siRNA (AVI)

CLSM-based video file showing Z-stacks of EGFP-Luc expressing MDA-MB-231 treated with ALSL5 auropolyplexes based on AF647-labeled siRNA and LPEI5 (AVI)

CLSM-based 3D reconstruction of EGFP-Luc expressing MDA-MB-231 treated with ALSL5 auropolyplexes based on AF647-labeled siRNA and LPEI5 (AVI)

CLSM-based video file showing Z-stacks of EGFP-Luc expressing MDA-MB-231 treated with BPEI polyplexes (as internal control) based on AF647-labeled siRNA and BPEI (AVI)

\section{AUTHOR INFORMATION}

\section{Corresponding Authors}

Manfred Ogris - Faculty of Life Sciences, Center of Pharmaceutical Sciences, Department of Pharmaceutical Chemistry, Laboratory of MacroMolecular Cancer Therapeutics (MMCT), University of Vienna, 1090 Vienna, Austria; Center for NanoScience (CeNS), Ludwig Maximilians University, 80539 Munich, Germany; Email: m.ogris@univie.ac.at

Haider Sami - Faculty of Life Sciences, Center of Pharmaceutical Sciences, Department of Pharmaceutical Chemistry, Laboratory of MacroMolecular Cancer Therapeutics (MMCT), University of Vienna, 1090 Vienna, Austria; 이이.org/0000-00025777-3472; Email: haider.sami@univie.ac.at

\section{Authors}

Alexander Taschauer - Faculty of Life Sciences, Center of Pharmaceutical Sciences, Department of Pharmaceutical Chemistry, Laboratory of MacroMolecular Cancer Therapeutics (MMCT), University of Vienna, 1090 Vienna, Austria

Wolfram Polzer - Faculty of Life Sciences, Center of Pharmaceutical Sciences, Department of Pharmaceutical Chemistry, Laboratory of MacroMolecular Cancer Therapeutics (MMCT), University of Vienna, 1090 Vienna, Austria

Stefan Pöschl - Faculty of Life Sciences, Center of Pharmaceutical Sciences, Department of Pharmaceutical Chemistry, Laboratory of MacroMolecular Cancer Therapeutics (MMCT), University of Vienna, 1090 Vienna, Austria

Slavica Metz - Faculty of Life Sciences, Center of Pharmaceutical Sciences, Department of Pharmaceutical Chemistry, Laboratory 
of MacroMolecular Cancer Therapeutics (MMCT), University of Vienna, 1090 Vienna, Austria

Nathalie Tepe - Department of Environmental Geosciences, University of Vienna, 1090 Vienna, Austria

Simon Decker - Faculty of Life Sciences, Center of Pharmaceutical Sciences, Department of Pharmaceutical Chemistry, Laboratory of MacroMolecular Cancer Therapeutics (MMCT), University of Vienna, 1090 Vienna, Austria

Norbert Cyran - Core Facility Cell Imaging and Ultrastructure Research (CIUS), University of Vienna, 1090 Vienna, Austria

Julia Scholda - Faculty of Life Sciences, Center of Pharmaceutical Sciences, Department of Pharmaceutical Chemistry, Laboratory of MacroMolecular Cancer Therapeutics (MMCT), University of Vienna, 1090 Vienna, Austria

Julia Maier - Faculty of Life Sciences, Center of Pharmaceutical Sciences, Department of Pharmaceutical Chemistry, Laboratory of MacroMolecular Cancer Therapeutics (MMCT), University of Vienna, 1090 Vienna, Austria

Hermann Bloß - Faculty of Life Sciences, Center of Pharmaceutical Sciences, Department of Pharmaceutical Chemistry, Laboratory of MacroMolecular Cancer Therapeutics (MMCT), University of Vienna, 1090 Vienna, Austria

Martina Anton - Institutes of Molecular Immunology and Experimental Oncology, Klinikum rechts der Isar, Technische Universität München, 81675 Munich, Germany

Thilo Hofmann - Department of Environmental Geosciences, University of Vienna, 1090 Vienna, Austria; (1) orcid.org/ 0000-0001-8929-6933

Complete contact information is available at: https://pubs.acs.org/10.1021/acsami.0c06608

\section{Notes}

The authors declare no competing financial interest.

\section{ACKNOWLEDGMENTS}

The research leading to these results has received support from the Innovative Medicines Initiative Joint Undertaking under grant agreement no. 115363, resources of which are composed of financial contribution from the European Union's Seventh Framework Programme (FP7/2007-2013) and EFPIA companies in kind contribution and the NANOBILD (Development and application of nanomaterials for multimodal medical imaging), an infrastructure grant of the Federal Ministry of Science, Research and Economy (BMBWF) from Austria. Antonia Geyer and Theresa Kittelmann are gratefully acknowledged for help in setting up the initial in vivo measurements. The oligonucleotides used in this work were supplied by GSK (GSK Medicines Research Centre, Stevenage, UK; synthesis by Glynn Williams and Jonathan Northall) as part of their contribution to the EU-funded IMI project COMPACT.

\section{REFERENCES}

(1) Morton, S. W.; Herlihy, K. P.; Shopsowitz, K. E.; Deng, Z. J.; Chu, K. S.; Bowerman, C. J.; Desimone, J. M.; Hammond, P. T. Scalable Manufacture of Built-to-Order Nanomedicine: SprayAssisted Layer-by-Layer Functionalization of Print Nanoparticles. Adv. Mater. 2013, 25, 4707-4713.

(2) Wei, A.; Mehtala, J. G.; Patri, A. K. Challenges and Opportunities in the Advancement of Nanomedicines. J. Controlled Release 2012, 164, 236-246.

(3) Gilleron, J.; Querbes, W.; Zeigerer, A.; Borodovsky, A.; Marsico, G.; Schubert, U.; Manygoats, K.; Seifert, S.; Andree, C.; Stöter, M.;
Epstein-Barash, H.; Zhang, L.; Koteliansky, V.; Fitzgerald, K.; Fava, E.; Bickle, M.; Kalaidzidis, Y.; Akinc, A.; Maier, M.; Zerial, M. ImageBased Analysis of Lipid Nanoparticle-Mediated Sirna Delivery, Intracellular Trafficking and Endosomal Escape. Nat. Biotechnol. 2013, 31, 638-646.

(4) Deng, Z. J.; Morton, S. W.; Ben-Akiva, E.; Dreaden, E. C.; Shopsowitz, K. E.; Hammond, P. T. Layer-by-Layer Nanoparticles for Systemic Codelivery of an Anticancer Drug and Sirna for Potential Triple-Negative Breast Cancer Treatment. ACS Nano 2013, 7, 95719584.

(5) Godfrey, C.; Desviat, L. R.; Smedsrød, B.; Piétri-Rouxel, F.; Denti, M. A.; Disterer, P.; Lorain, S.; Nogales-Gadea, G.; Sardone, V.; Anwar, R.; El Andaloussi, S.; Lehto, T.; Khoo, B.; Brolin, C.; van Roon-Mom, W. M.; Goyenvalle, A.; Aartsma-Rus, A.; ArechavalaGomeza, V. Delivery Is Key: Lessons Learnt from Developing SpliceSwitching Antisense Therapies. EMBO Mol. Med. 2017, 9, 545-557.

(6) Elbakry, A.; Zaky, A.; Liebl, R.; Rachel, R.; Goepferich, A.; Breunig, M. Layer-by-Layer Assembled Gold Nanoparticles for Sirna Delivery. Nano Lett. 2009, 9, 2059-2064.

(7) Correa, S.; Choi, K. Y.; Dreaden, E. C.; Renggli, K.; Shi, A.; Gu, L.; Shopsowitz, K. E.; Quadir, M. A.; Ben-Akiva, E.; Hammond, P. T. Highly Scalable, Closed-Loop Synthesis of Drug-Loaded, Layer-byLayer Nanoparticles. Adv. Funct. Mater. 2016, 26, 991-1003.

(8) Tan, Y. F.; Mundargi, R. C.; Chen, M. H. A.; Lessig, J.; Neu, B.; Venkatraman, S. S.; Wong, T. T. Layer-by-Layer Nanoparticles as an Efficient Sirna Delivery Vehicle for Sparc Silencing. Small 2014, 10, 1790-1798.

(9) Wurster, E.-C.; Liebl, R.; Michaelis, S.; Robelek, R.; Wastl, D. S.; Giessibl, F. J.; Goepferich, A.; Breunig, M. Oligolayer-Coated Nanoparticles: Impact of Surface Topography at the Nanobio Interface. ACS Appl. Mater. Interfaces 2015, 7, 7891-7900.

(10) Yin, H.; Kanasty, R. L.; Eltoukhy, A. A.; Vegas, A. J.; Dorkin, J. R; Anderson, D. G. Non-Viral Vectors for Gene-Based Therapy. Nat. Rev. Genet. 2014, 15, 541-555.

(11) Kanasty, R.; Dorkin, J. R.; Vegas, A.; Anderson, D. Delivery Materials for Sirna Therapeutics. Nat. Mater. 2013, 12, 967-977.

(12) Barnaby, S. N.; Lee, A.; Mirkin, C. A. Probing the Inherent Stability of Sirna Immobilized on Nanoparticle Constructs. Proc. Natl. Acad. Sci. U. S. A. 2014, 111, 9739-9744.

(13) Rosi, N. L.; Giljohann, D. A.; Thaxton, C. S.; Lytton-Jean, A. K. R.; Han, M. S.; Mirkin, C. A. Oligonucleotide-Modified Gold Nanoparticles for Intracellular Gene Regulation. Science 2006, 312, 1027-1030.

(14) Taschauer, A.; Geyer, A.; Gehrig, S.; Maier, J.; Sami, H.; Ogris, M. Up-Scaled Synthesis and Characterization of Nonviral Gene Delivery Particles for Transient in Vitro and in Vivo Transgene Expression. Hum. Gene Ther: Methods. 2016, 27, 87-97.

(15) Lächelt, U.; Wagner, E. Nucleic Acid Therapeutics Using Polyplexes: A Journey of 50 Years (and Beyond). Chem. Rev. 2015, $115,11043-11078$.

(16) Höbel, S.; Aigner, A. Polyethylenimines for Sirna and Mirna Delivery in Vivo. WIREs Nanomed. Nanobiotechnol. 2013, 5, 484-501.

(17) Fahrmeir, J.; Gunther, M.; Tietze, N.; Wagner, E.; Ogris, M. Electrophoretic Purification of Tumor-Targeted PolyethylenimineBased Polyplexes Reduces Toxic Side Effects in Vivo. J. Controlled Release 2007, 122, 236-245.

(18) Bonnet, M.-E.; Erbacher, P.; Bolcato-Bellemin, A.-L. Systemic Delivery of DNA or Sirna Mediated by Linear Polyethylenimine (LPei) Does Not Induce an Inflammatory Response. Pharm. Res. 2008, 25, 2972-2982.

(19) Di Gioia, S.; Conese, M. Polyethylenimine-Mediated Gene Delivery to the Lung and Therapeutic Applications. Drug Des., Dev. Ther. 2008, 2, 163-188.

(20) Buscail, L.; Bournet, B.; Vernejoul, F.; Cambois, G.; Lulka, H.; Hanoun, N.; Dufresne, M.; Meulle, A.; Vignolle-Vidoni, A.; Ligat, L.; Saint-Laurent, N.; Pont, F.; Dejean, S.; Gayral, M.; Martins, F.; Torrisani, J.; Barbey, O.; Gross, F.; Guimbaud, R.; Otal, P.; Lopez, F.; Tiraby, G.; Cordelier, P. First-in-Man Phase 1 Clinical Trial of Gene 
Therapy for Advanced Pancreatic Cancer: Safety, Biodistribution, and Preliminary Clinical Findings. Mol. Ther. 2015, 23, 779-789.

(21) Meleshko, A. N.; Petrovskaya, N. A.; Savelyeva, N.; Vashkevich, K. P.; Doronina, S. N.; Sachivko, N. V. Phase I Clinical Trial of Idiotypic DNA Vaccine Administered as a Complex with Polyethylenimine to Patients with B-Cell Lymphoma. Hum. Vaccines Immunother. 2017, 13, 1-6.

(22) Anchiano Therapeutics Israel Ltd. Codex: Study of Inodiftagene Vixteplasmid (Bc-819) in Unresponsive Nmibc. https://ClinicalTrials. gov/show/NCT03719300: 2018.

(23) Rome, C.; Gravier, J.; Morille, M.; Divita, G.; Bolcato-Bellemin, A.-L.; Josserand, V.; Coll, J.-L. Near-Infrared Optical Imaging of Nucleic Acid Nanocarriers in Vivo. Methods Mol. Biol. 2019, 1943, 347-363.

(24) Geyer, A.; Lorenzer, C.; Gehrig, S.; Simlinger, M.; Winkler, J.; Sami, H.; Ogris, M. Fluorescence- and Computed Tomography for Assessing the Biodistribution of Sirna after Intratracheal Application in Mice. Int. J. Pharm. 2017, 525, 359-366.

(25) Thanki, K.; van Eetvelde, D.; Geyer, A.; Fraire, J.; Hendrix, R.; Van Eygen, H.; Putteman, E.; Sami, H.; de Souza Carvalho-Wodarz, C.; Franzyk, H.; Nielsen, H. M.; Braeckmans, K.; Lehr, C.-M.; Ogris, M.; Foged, C. Mechanistic Profiling of the Release Kinetics of Sirna from Lipidoid-Polymer Hybrid Nanoparticles in Vitro and in Vivo after Pulmonary Administration. J. Controlled Release 2019, 310, 8293.

(26) Rehman, Z. u.; Hoekstra, D.; Zuhorn, I. S. Mechanism of Polyplex- and Lipoplex-Mediated Delivery of Nucleic Acids: RealTime Visualization of Transient Membrane Destabilization without Endosomal Lysis. ACS Nano 2013, 7, 3767-3777.

(27) Rödl, W.; Schaffert, D.; Wagner, E.; Ogris, M. Synthesis of Polyethylenimine-Based Nanocarriers for Systemic Tumor Targeting of Nucleic Acids. Methods Mol. Biol. 2013, 948, 105-120.

(28) Ungaro, F.; De Rosa, G.; Miro, A.; Quaglia, F. Spectrophotometric Determination of Polyethylenimine in the Presence of an Oligonucleotide for the Characterization of Controlled Release Formulations. J. Pharm. Biomed. Anal. 2003, 31, 143-149.

(29) Schaffert, D.; Kiss, M.; Rödl, W.; Shir, A.; Levitzki, A.; Ogris, M.; Wagner, E. Poly(I:C)-Mediated Tumor Growth Suppression in Egf-Receptor Overexpressing Tumors Using Egf-Polyethylene GlycolLinear Polyethylenimine as Carrier. Pharm. Res. 2011, 28, 731-741.

(30) Frens, G. Controlled Nucleation for the Regulation of the Particle Size in Monodisperse Gold Suspensions. Nat. Phys. Sci. 1973, 241, 20.

(31) Wurster, E. C.; Elbakry, A.; Göpferich, A.; Breunig, M. Layerby-Layer Assembled Gold Nanoparticles for the Delivery of Nucleic Acids. Methods Mol. Biol. 2013, 948, 171-182.

(32) Su, B.; Cengizeroglu, A.; Farkasova, K.; Viola, J. R.; Anton, M.; Ellwart, J. W.; Haase, R.; Wagner, E.; Ogris, M. Systemic Tnf $\alpha$ Gene Therapy Synergizes with Liposomal Doxorubicine in the Treatment of Metastatic Cancer. Mol. Ther. 2013, 21, 300-308.

(33) Müller, K.; Ogris, M.; Sami, H. Firefly Luciferase-Based Reporter Gene Assay for Investigating Nanoparticle-Mediated Nucleic Acid Delivery. Methods Mol. Biol. 2019, 1943, 227-239.

(34) Templeton, A. C.; Hostetler, M. J.; Warmoth, E. K.; Chen, S.; Hartshorn, C. M.; Krishnamurthy, V. M.; Forbes, M. D. E.; Murray, R. W. Gateway Reactions to Diverse, Polyfunctional MonolayerProtected Gold Clusters. J. Am. Chem. Soc. 1998, 120, 4845-4849.

(35) Curtis, K. A.; Miller, D.; Millard, P.; Basu, S.; Horkay, F.; Chandran, P. L. Unusual Salt and $\mathrm{Ph}$ Induced Changes in Polyethylenimine Solutions. PLoS One 2016, 11, No. e0158147.

(36) Chen, H.; Paholak, H.; Ito, M.; Sansanaphongpricha, K.; Qian, W.; Che, Y.; Sun, D. 'Living' Pegylation on Gold Nanoparticles to Optimize Cancer Cell Uptake by Controlling Targeting Ligand and Charge Densities. Nanotechnology 2013, 24, 355101.

(37) Levin, C. S.; Bishnoi, S. W.; Grady, N. K.; Halas, N. J. Determining the Conformation of Thiolated Poly(Ethylene Glycol) on Au Nanoshells by Surface-Enhanced Raman Scattering Spectroscopic Assay. Anal. Chem. 2006, 78, 3277-3281.
(38) Thomas, M.; Klibanov, A. M. Conjugation to Gold Nanoparticles Enhances Polyethylenimine's Transfer of Plasmid DNA into Mammalian Cells. Proc. Natl. Acad. Sci. U. S. A. 2003, 100, 91389143.

(39) Boeckle, S.; von Gersdorff, K.; van der Piepen, S.; Culmsee, C.; Wagner, E.; Ogris, M. Purification of Polyethylenimine Polyplexes Highlights the Role of Free Polycations in Gene Transfer. J. Gene Med. 2004, 6, 1102-1111.

(40) Benjaminsen, R. V.; Mattebjerg, M. A.; Henriksen, J. R.; Moghimi, S. M.; Andresen, T. L. The Possible "Proton Sponge" Effect of Polyethylenimine (Pei) Does Not Include Change in Lysosomal Ph. Mol. Ther. 2013, 21, 149-157.

(41) von Gersdorff, K.; Sanders, N. N.; Vandenbroucke, R.; De Smedt, S. C.; Wagner, E.; Ogris, M. The Internalization Route Resulting in Successful Gene Expression Depends on Both Cell Line and Polyethylenimine Polyplex Type. Mol. Ther. 2006, 14, 745-753.

(42) Jokerst, J. V.; Lobovkina, T.; Zare, R. N.; Gambhir, S. S. Nanoparticle Pegylation for Imaging and Therapy. Nanomedicine 2011, 6, 715-728.

(43) Giljohann, D. A.; Seferos, D. S.; Prigodich, A. E.; Patel, P. C.; Mirkin, C. A. Gene Regulation with Polyvalent Sirna-Nanoparticle Conjugates. J. Am. Chem. Soc. 2009, 131, 2072-2073.

(44) Melamed, J. R.; Kreuzberger, N. L.; Goyal, R.; Day, E. S. Spherical Nucleic Acid Architecture Can Improve the Efficacy of Polycation-Mediated Sirna Delivery. Mol. Ther. Nucleic Acids 2018, 12, 207-219.

(45) Kopatz, I.; Remy, J.-S.; Behr, J.-P. A Model for Non-Viral Gene Delivery: Through Syndecan Adhesion Molecules and Powered by Actin. J. Gene Med. 2004, 6, 769-776.

(46) Patel, P. C.; Giljohann, D. A.; Daniel, W. L.; Zheng, D.; Prigodich, A. E.; Mirkin, C. A. Scavenger Receptors Mediate Cellular Uptake of Polyvalent Oligonucleotide-Functionalized Gold Nanoparticles. Bioconjugate Chem. 2010, 21, 2250-2256.

(47) Juliano, R. L.; Ming, X.; Nakagawa, O. Cellular Uptake and Intracellular Trafficking of Antisense and Sirna Oligonucleotides. Bioconjugate Chem. 2012, 23, 147-157.

(48) Bus, T.; Traeger, A.; Schubert, U. S. The Great Escape: How Cationic Polyplexes Overcome the Endosomal Barrier. J. Mater. Chem. B 2018, 6, 6904-6918.

(49) Kichler, A.; Leborgne, C.; Danos, O. Dilution of Reporter Gene with Stuffer DNA Does Not Alter the Transfection Efficiency of Polyethylenimines. J. Gene Med. 2005, 7, 1459-1467.

(50) Merkel, O. M.; Beyerle, A.; Librizzi, D.; Pfestroff, A.; Behr, T. M.; Sproat, B.; Barth, P. J.; Kissel, T. Nonviral Sirna Delivery to the Lung: Investigation of Peg-Pei Polyplexes and Their in Vivo Performance. Mol. Pharmaceutics 2009, 6, 1246-1260.

(51) Klutz, K.; Schaffert, D.; Willhauck, M. J.; Grünwald, G. K.; Haase, R.; Wunderlich, N.; Zach, C.; Gildehaus, F. J.; SenekowitschSchmidtke, R.; Göke, B.; Wagner, E.; Ogris, M.; Spitzweg, C. Epidermal Growth Factor Receptor-Targeted ${ }^{131}$ I-Therapy of Liver Cancer Following Systemic Delivery of the Sodium Iodide Symporter Gene. Mol. Ther. 2011, 19, 676-685.

(52) Schäfer, A.; Pahnke, A.; Schaffert, D.; van Weerden, W. M.; de Ridder, C. M. A.; Rödl, W.; Vetter, A.; Spitzweg, C.; Kraaij, R.; Wagner, E.; Ogris, M. Disconnecting the Yin and Yang Relation of Epidermal Growth Factor Receptor (Egfr)-Mediated Delivery: A Fully Synthetic, Egfr-Targeted Gene Transfer System Avoiding Receptor Activation. Hum. Gene Ther. 2011, 22, 1463-1473.

(53) Feldmann, D. P.; Merkel, O. M. The Advantages of Pulmonary Delivery of Therapeutic Sirna. Ther. Delivery 2015, 6, 407-409.

(54) Geyer, A.; Taschauer, A.; Alioglu, F.; Anton, M.; Maier, J.; Drothler, E.; Simlinger, M.; Yavuz, S.; Sami, H.; Ogris, M. Multimodal Fluorescence and Bioluminescence Imaging Reveals Transfection Potential of Intratracheally Administered Polyplexes for Breast Cancer Lung Metastases. Hum. Gene Ther. 2017, 28, 1202-1213.

(55) Moschos, S. A.; Frick, M.; Taylor, B.; Turnpenny, P.; Graves, H.; Spink, K. G.; Brady, K.; Lamb, D.; Collins, D.; Rockel, T. D.; Weber, M.; Lazari, O.; Perez-Tosar, L.; Fancy, S. A.; Lapthorn, C.; Green, M. X.; Evans, S.; Selby, M.; Jones, G.; Jones, L.; Kearney, S.; 
Mechiche, H.; Gikunju, D.; Subramanian, R.; Uhlmann, E.; Jurk, M.; Vollmer, J.; Ciaramella, G.; Yeadon, M. Uptake, Efficacy, and Systemic Distribution of Naked, Inhaled Short Interfering Rna (Sirna) and Locked Nucleic Acid (Lna) Antisense. Mol. Ther. 2011, 19, 2163-2168.

(56) Schleh, C.; Holzwarth, U.; Hirn, S.; Wenk, A.; Simonelli, F.; Schäffler, M.; Möller, W.; Gibson, N.; Kreyling, W. G. Biodistribution of Inhaled Gold Nanoparticles in Mice and the Influence of Surfactant Protein D. J. Aerosol. Med. Pulm. Drug Delivery 2013, 26, 24-30.

(57) Sadauskas, E.; Jacobsen, N. R.; Danscher, G.; Stoltenberg, M.; Vogel, U.; Larsen, A.; Kreyling, W.; Wallin, H. Biodistribution of Gold Nanoparticles in Mouse Lung Following Intratracheal Instillation. Chem. Cent. J. 2009, 3, 16.

(58) Troiber, C.; Kasper, J. C.; Milani, S.; Scheible, M.; Martin, I.; Schaubhut, F.; Küchler, S.; Rädler, J.; Simmel, F. C.; Friess, W.; Wagner, E. Comparison of Four Different Particle Sizing Methods for Sirna Polyplex Characterization. Eur. J. Pharm. Biopharm. 2013, 84, 255-264.

(59) Anchiano Therapeutics Israel Ltd. Phase 2b, Trial of Intravesical Dta-H19/Pei in Patients with Intermediate-Risk Superficial Bladder Cancer; https://ClinicalTrials.gov/show/NCT00595088: 2008.

(60) Anchiano Therapeutics Israel Ltd. Pilot Study of Bc-819/Pei and Bcg in Patients with Superficial Transitional Cell Bladder Carcinoma;. https://ClinicalTrials.gov/show/NCT01878188: 2013.

(61) Sung, J. H.; Ji, J. H.; Park, J. D.; Song, M. Y.; Song, K. S.; Ryu, H. R.; Yoon, J. U.; Jeon, K. S.; Jeong, J.; Han, B. S.; Chung, Y. H.; Chang, H. K.; Lee, J. H.; Kim, D. W.; Kelman, B. J.; Yu, I. J. Subchronic Inhalation Toxicity of Gold Nanoparticles. Part. Fibre Toxicol. 2011, 8, 16.

(62) Sanders, N.; Rudolph, C.; Braeckmans, K.; De Smedt, S. C.; Demeester, J. Extracellular Barriers in Respiratory Gene Therapy. Adv. Drug Delivery Rev. 2009, 61, 115-127.

(63) Taschauer, A.; Polzer, W.; Alioglu, F.; Billerhart, M.; Decker, S.; Kittelmann, T.; Geppl, E.; Elmenofi, S.; Zehl, M.; Urban, E.; Sami, H.; Ogris, M. Peptide-Targeted Polyplexes for Aerosol-Mediated Gene Delivery to Cd49f-Overexpressing Tumor Lesions in Lung. Mol. Ther. Nucleic Acids 2019, 18, 774-786. 